Draft VERSION NOVEMBER 3, 2018

Preprint typeset using $\mathrm{IAT}_{\mathrm{E}} \mathrm{X}$ style emulateapj v. 12/16/11

\title{
TWO POPULATIONS OF MOLECULAR CLOUDS IN THE ANTENNAE GALAXIES
}

\author{
Lisa H. Wei ${ }^{1}$, Eric Keto ${ }^{1}$, And Luis C. $\mathrm{Ho}^{2}$ \\ Draft version November 3, 2018
}

\begin{abstract}
Super star clusters - extremely massive clusters found predominately in starburst environments are essential building blocks in the formation of galaxies and thought to dominate star formation in the high-redshift universe. However, the transformation from molecular gas into these ultra-compact star clusters is not well understood. To study this process, we used the Submillimeter Array and the Plateau de Bure Interferometer to obtain high angular resolution $\left(\sim 1^{\prime \prime} 5\right.$ or $\left.160 \mathrm{pc}\right)$ images of the Antennae overlap region in $\mathrm{CO}(2-1)$ to search for the molecular progenitors of the super star clusters. We resolve the molecular gas distribution into a large number of clouds, extending the differential cloud mass function down to a $5 \sigma$ completeness limit of $3.8 \times 10^{5} M_{\odot}$. We identify a distinct break in the mass function around $\log M_{\mathrm{mol}} / M_{\odot} \approx 6.5$, which separates the molecular clouds into two distinct populations. The smaller, less massive clouds reside in more quiescent areas in the region, while the larger, more massive clouds cluster around regions of intense star formation. A broken power-law fit to the mass function yields slopes of $\alpha=-1.39 \pm 0.10$ and $\alpha=-1.44 \pm 0.14$ for the low- and high-mass cloud population, well-matched to the mass function found for super star clusters in the Antennae galaxies. We find large velocity gradients and velocity dispersions at the locations of intense star formation, suggestive of compressive shocks. It is likely that these environmental factors contribute to the formation of the observed massive molecular clouds and super star clusters in the Antennae galaxies.

Subject headings: galaxies: individual (NGC 4038/4039) — galaxies: ISM — galaxies: starburst — galaxies: star clusters
\end{abstract}

\section{INTRODUCTION}

In areas of lower extinction between the dust lanes of starbursts, observations by the Hubble Space Telescope $(H S T)$ have revealed an important connection between the starburst phenomenon and a class of objects known as "super star clusters" (SSCs; Larsen 2010; Portegies Zwart et al. 2010). SSCs represent the most extreme mode of star formation known. Aside from their extraordinary luminosities, which range from 1-100 times that of the R136 cluster of 30 Doradus in the Large Magellanic Cloud (Holtzman et al. 1992; Whitmore 2003; Larsen \& Richtler 2004), these clusters are exceptionally compact (radii $\leq 1-5$ pc; Larsen 2010; Portegies Zwart et al. 2010) and young ( $\lesssim$ few $-50 \mathrm{Myr}$; Portegies Zwart et al. 2010). Estimates based on high-resolution spectroscopy and population synthesis models suggest that cluster masses range from $10^{4}$ to $10^{6} M_{\odot}$ (e.g., Ho \& Filippenko 1996a,b; Zhang \& Fall 1999; Mengel et al. 2002; Larsen \& Richtler 2004; Melo et al. 2005; McCrady \& Graham 2007). SSCs comprise a significant fraction of the UV, $\mathrm{H} \alpha$, and $B$-band light emitted by young stars in starburst regions (Barth et al. 1995; Meurer et al. 1995; Maoz et al. 1996; Zepf et al. 1999; Fall et al. 2005). This suggests that in these regions SSCs are the basic building blocks of star formation (Ho 1997).

The similarity in size and mass of the SSCs and globular clusters suggests that the SSCs might be presentday analogs of young globular clusters (Holtzman et al. 1992; McCrady \& Graham 2007). Previously thought

\footnotetext{
${ }^{1}$ Harvard-Smithsonian Center for Astrophysics, 60 Garden Street, Cambridge, MA 02138

2 The Observatories of the Carnegie Institution for Science, 813 Santa Barbara Street, Pasadena, CA 91101
}

to form strictly in the primordial epoch of galaxy evolution, globular clusters may in fact be forming in the current epoch as SSCs in starburst environments (Ashman \& Zepf 2001).

The formation of SSCs, however, is not well understood. Some key questions include: How is the mass of the precursor molecular cloud, equivalent to a giant molecular cloud (GMC), converted to stars quickly and efficiently enough for the star cluster to remain bound? How are the conditions in starbursts different from "ordinary" star formation? Are there other distinguishing factors that differentiate progenitor clouds of SSCs from normal GMCs?

Recent studies suggest that it is high environmental pressure that increases the gas density and ultimately leads to the formation of massive star clusters (e.g., Ashman \& Zepf 2001; Herrera et al. 2011). One hypothesis suggests that a sudden galactic-scale increase in external pressure, possibly from shock compression associated with mergers, crushes entire GMCs rapidly and efficiently, forming individual SSCs (Jog \& Solomon 1992; Ashman \& Zepf 2001). CO(2-1) line observations of M82 with the Owens Valley Millimeter Array (OVRO) with 17 pc spatial resolution by Keto et al. (2005) support this, showing that the mass spectrum of GMCs in the starburst region is indistinguishable from the upper range of the mass spectrum of SSCs in M82 (Melo et al. 2005). These observations further show that the clouds with active star formation are in supersonic compression, with both lateral and line-of-sight velocity gradients within the individual clouds.

In this hypothesis, the compression itself is created by the rapid ionization of the interstellar medium (ISM) 
surrounding the progenitor GMCs. The initial ionization may be caused by the dissipation of kinetic energy in the diffuse ISM of colliding galaxies. The burst of massive star formation that follows then releases enough energy to further propagate the ionization and compress nearby molecular clouds. This hypothesis for ionizationdriven implosion draws a fascinating connection between the present-day SSCs and the ancient globular clusters in that the globular clusters may also have formed in clouds compressed by rapid ionization in the epoch of reionization in the primordial universe at $z \approx 6$ (Cen 2001; van den Bergh 2001).

Alternatively, SSCs may form in the high-pressure environment of the centers of super-giant molecular complexes $\left(M \geq 10^{7} M_{\odot}\right.$; Harris \& Pudritz 1994; Wilson et al. 2003). In this hypothesis, cores that are the size of GMCs, but are within larger molecular complexes, are slowly compressed (on a crossing time) by the pressure of the overlying molecular gas. Cluster formation proceeds as a scaled-up version of normal star formation in our own Galaxy (Elmegreen \& Efremov 1997). In support of this alternative, $\mathrm{CO}(1-0)$ line observations of the Antennae galaxies show huge concentrations of molecular gas that could be interpreted as super-giant molecular complexes (Wilson et al. 2003). Yet because the angular resolution of these observations $\left(33^{\prime \prime} 2 \times 4^{\prime \prime} .9 ; \sim 420 \mathrm{pc}\right.$ at the adopted distance of the $22 \mathrm{Mpc}$ ) is insufficient to resolve individual GMCs, the question remains whether the appearance of the extraordinary massive clouds is simply the result of limited spatial resolution.

In this paper we revisit the Antennae galaxies, NGC 4038 and NGC 4039, with higher resolution imaging of the molecular gas to study the formation of SSCs. As one of the most spectacular starbursts in the local universe, the Antennae has been studied in great detail over the last several decades in various wavelengths, ranging from the X-rays to the $\mathrm{mm} / \mathrm{cm}$ regime, and everything in between (e.g., Mirabel et al. 1998; Neff \& Ulvestad 2000; Wilson et al. 2000, 2003; Fabbiano et al. 2001, 2003; Hibbard et al. 2001, 2005; Zhang et al. 2001; Kilgard et al. 2002; Mengel et al. 2002; Zezas et al. 2002; Zezas \& Fabbiano 2002; Fall et al. 2005). It is also one of the beststudied SSC populations (Whitmore \& Schweizer 1995; Whitmore et al. 1999, 2010).

The overlap region of the Antennae, where NGC 4038 and NGC 4039 collide, is one of the most intensely and violently star-forming regions in the nearby universe (Evans et al. 1997; Mirabel et al. 1998; Gao et al. 2001; Gao 2008; Brandl et al. 2009; Klaas et al. 2010; Zhang et al. 2010). The star formation rate (SFR) for the entire NGC 4038/39 system is high, estimated to be between 6.6-20 $M_{\odot} \mathrm{yr}^{-1}$ (Zhang et al. 2001; Brandl et al. 2009). However, most of the star formation occurs in the overlap region, with SFRs of $0.7-2.0 M_{\odot} \mathrm{yr}^{-1}$ for individual starforming regions on size scales of $500 \times 1200 \mathrm{pc}^{2}$ (Brandl et al. 2009). Recent studies also find intriguing evidence for shock-driven star formation (Gao et al. 2001; Zhang et al. 2010), in agreement with the Jog \& Solomon model. Detailed population synthesis analysis of the clusters in these regions finds the median age of the clusters to be some of the youngest in the system, $\lesssim 10 \mathrm{Myr}$ in many cases (e.g., Snijders et al. 2007; Zhang et al. 2010).

In this paper, Section 1 comprises this introduction. In

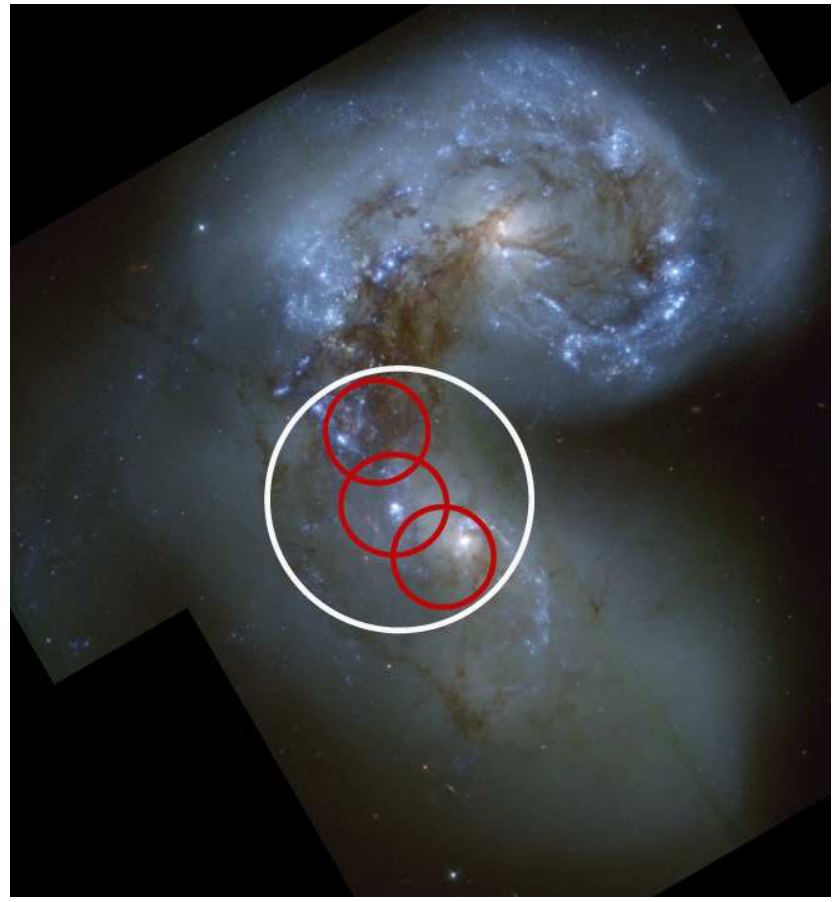

FIG. 1.- : Three-color image of the Antennae from the HST Advanced Camera of Surveys (ACS), using data taken by Whitmore et al. (2010). Filters used to create the image are F435W (blue), F550M (green), and F814W (red). The image has dimensions of $160^{\prime \prime} \times 195^{\prime \prime}$, and it is displayed using an arcsinh stretch (Lupton et al. 2004). The white circle shows the primary beam of our SMA observations, and red circles show our PdBI pointings. See Table 1 for more information.

Section 2 we present new, high-resolution $\mathrm{CO}(2-1)$ data from the Submillimeter Array (SMA; Ho et al. 2004) and the IRAM Plateau de Bure Interferometer ${ }^{3}$ (PdBI). Section 3 presents some basic properties of the molecular gas in the observed region and discusses the implications. In Section 4 we compare the distribution of molecular gas mass, velocity, and velocity dispersion with star formation tracers. In Section 5, we summarize our results. Throughout this paper we adopt a distance of $22 \mathrm{Mpc}$ for the Antennae (Schweizer et al. 2008).

\section{OBSERVATIONS}

\subsection{The Submillimeter Array}

The SMA observations were taken in May 2008 and February 2011 in the compact and extended array configurations, respectively, with a single pointing with a phase center $(\alpha, \delta)_{\mathrm{J} 2000}=\left(12^{\mathrm{h}} 01^{\mathrm{m}} 54^{\mathrm{s}} .70,-18^{\circ} 53^{\prime} 05^{\prime \prime} \cdot 0\right)$. Figure 1 shows our spatial coverage of the overlap region $^{4}$. At $230 \mathrm{GHz}$, the primary beam of the SMA covers $\sim 54^{\prime \prime}$. Baselines ranged from 16 to 139 meters (compact array) and 44 and 226 meters (extended array). Typical system temperatures ranged between 100 and $300 \mathrm{~K}$ for both observations. The correlator was tuned to the frequency of $\mathrm{CO}(2-1)$ at $230.53799 \mathrm{GHz}$, with Doppler

3 IRAM is supported by INSU/CNRS (France), MPG (Germany) and IGN (Spain).

4 Based on observations made with the NASA/ESA Hubble Space Telescope, obtained from the data archive at the Space Telescope Science Institute. STScI is operated by the Association of Universities for Research in Astronomy, Inc. under NASA contract NAS 5-26555. 
tracking accounting for the recessional velocity of the Antennae galaxies. For the May 2008 track, the correlator was configured to cover a $2 \mathrm{GHz}$ bandwidth with 24 overlapping windows each $104 \mathrm{MHz}$ in width, with a spectral resolution of $0.4 \mathrm{MHz}\left(\sim 0.5 \mathrm{~km} \mathrm{~s}^{-1}\right.$ channels $)$. The February 2011 track was configured slightly differently due to the bandwidth doubling upgrade by the SMA in 2009 , covering $4 \mathrm{GHz}$ with 48 overlapping windows each $104 \mathrm{MHz}$ in width, with a spectral resolution of $0.8 \mathrm{MHz}$ $\left(\sim 1 \mathrm{~km} \mathrm{~s}^{-1}\right.$ channels).

The SMA data were reduced using the MIR data reduction package developed at OVRO and the SMA. Data were flagged for bad channels, antennas, weather, and pointing. Bandpass calibration was done on a bright quasar, 3C 454.3 for the May 2008 observation and 3C 84 for the February 2011 observation. We performed atmospheric phase calibrations with $1127-189$ and $1256-057$ every 10 minutes. Absolute flux calibration was done using Callisto and Titan. The channels were Hanning smoothed and averaged to achieve a final resolution of $4.9 \mathrm{~km} \mathrm{~s}^{-1}$.

\subsection{Plateau de Bure Interferometer}

The PdBI observations were taken between February and April of 2006 in the B and C arrays, respectively. Because of the smaller $\left(\sim 21^{\prime \prime}\right)$ primary beam of the PdBI 15 meter antennas, three separate pointings were obtained to cover approximately the same area of the overlap region as the SMA primary beam at $230 \mathrm{GHz}$ (Figure 1 \& Table 1). Each pointing was observed with a single $\sim 7$ hour track at the PdBI. Baselines ranged from 24 to 82 meters in the $\mathrm{C}$ array and 40 to 280 meters in the B array. Typical system temperatures ranged from 300 to $1000 \mathrm{~K}$ due to the low elevation of the Antennae for the PdBI. The correlator was configured to cover the $\mathrm{CO}(2-1)$ line with four windows each $160 \mathrm{MHz}$ in width, with 160 channels in each window with spectral resolution of $1.25 \mathrm{MHz}\left(\sim 1.6 \mathrm{~km} \mathrm{~s}^{-1}\right.$ channels).

The PdBI data were reduced using the GILDAS CLIC package. Bandpass and absolute flux calibration were done using $3 \mathrm{C} 273$, and the atmospheric phase was calibrated using 1124-018 every 20 minutes. The channels were Hanning smoothed and averaged to achieve a final resolution of $4.9 \mathrm{~km} \mathrm{~s}^{-1}$.

\subsection{Imaging}

To recover the more extended flux from the shorter spacings and increase the signal-to-noise ratio, we combine the high-resolution SMA and PdBI data together with the SMA compact array observations in the $(u, v)$ plane. After reduction, the PdBI $(u, v)$ data were saved as FITS files, then converted into the MIRIAD format using the task fits. Header information including telescope name, longitude, latitude, and channel resolution were manually put into the PdBI MIRIAD datasets. We combined the data with the MIRIAD software package (Sault et al. 1995), using natural weighting and an additional weighting in inverse proportion to the noise as estimated by the system temperature. The data cube was cleaned to a cutoff of $2 \sigma_{\text {chan }}$ in the residual image. The synthesized beam of the combined SMA+PdBI image is $3^{\prime \prime} .3 \times 1^{\prime \prime} .5$, with a position angle of $41^{\circ}$. The rms in a single $4.9 \mathrm{~km} \mathrm{~s}^{-1}$ channel $\left(\sigma_{\text {chan }}\right)$ is $30 \mathrm{mJy}_{\text {beam }}{ }^{-1}$.
TABLE 1

Telescope Pointing Centers

\begin{tabular}{lc}
\hline \hline Position & $\begin{array}{c}\text { Phase Center } \\
\left(\alpha_{2000}, \delta_{2000}\right)\end{array}$ \\
\hline SMA Comp & $12^{\mathrm{h}} 01^{\mathrm{m}} 54^{\mathrm{s}} .70,-18^{\circ} 53^{\prime} 05^{\prime \prime} 0$ \\
SMA Ext & $12^{\mathrm{h}} 01^{\mathrm{m}} 54^{\mathrm{s}} .70,-18^{\circ} 53^{\prime} 05^{\prime \prime} 0$ \\
PdBI A & $12^{\mathrm{h}} 01^{\mathrm{m}} 55^{\mathrm{s}} .00,-18^{\circ} 52^{\prime} 50^{\prime \prime} 0$ \\
PdBI B & $12^{\mathrm{h}} 01^{\mathrm{m}} 54^{\mathrm{s}} .70,-18^{\circ} 53^{\prime} 05^{\prime \prime} 0$ \\
PdBI C & $12^{\mathrm{h}} 01^{\mathrm{m}} 54^{\mathrm{s}} .00,-18^{\circ} 53^{\prime} 15^{\prime \prime} 0$ \\
\hline
\end{tabular}

Velocity-integrated emission (zeroth moment), intensity-weighted velocity (first moment), and intensityweighted dispersion (second moment) maps were made from a masked data cube. The masks were made by smoothing the original cube to a resolution of $6^{\prime \prime}$ and masking emission that was less than $2 \sigma$ or did not cover two contiguous channels. Figure 2 shows the velocity-integrated $\mathrm{CO}(2-1)$ emission of our combined PdBI+SMA observations of the overlap region.

\section{PROPERTIES OF THE MOLECULAR GAS}

\subsection{Finding Clouds}

To extract individual clouds from our data cubes, we utilize the algorithm clumpfind by Williams et al. (1994) on our data cube where the PdBI and SMA primary beams overlap. clumpfind contours the input data cube in steps that are integer multiples of the input channel rms and identifies clouds as emission peaks separated by more than one contour. The extent of each cloud is determined by following each peak down to the first (lowest) contour. Multi-peaked emission is separated by a "friends-of-friends" algorithm where pixels adjacent to the peak and subsequent adjacent pixels are identified as related.

The default contour and detection thresholds recommended for clumpfind by Williams et al. (1994) are $2 \sigma_{\text {chan }}$. We tested three different detection thresholds $\left(2,2.5\right.$, and $3 \sigma_{\text {chan }}$ while holding the contour threshold constant at $\left.2 \sigma_{\text {chan }}\right)$. However, we find that most of the results do not depend strongly on the choice of detection threshold. We discuss this in more detail with the individual results. We set the clumpfind search parameters to contour at twice the channel rms $\left(2 \sigma_{\text {chan }}\right)$ with the detection threshold at $3 \sigma_{\text {chan }}$ to detect the faintest clouds with a higher confidence level and better avoid spurious detections of low-mass clouds.

\subsection{Fluxes and Mass Estimates}

We estimate the mass of each cloud from its CO luminosity following Solomon et al. (1992):

$\frac{L_{\mathrm{CO}}}{\mathrm{K} \mathrm{km} \mathrm{s}^{-1} \mathrm{pc}^{2}}=3.25 \times 10^{7} \nu_{\text {rest }}^{-2}(1+z)^{-1}\left(\frac{D_{L}}{\mathrm{Mpc}}\right)^{2}\left(\frac{F_{\mathrm{CO}}^{\prime}}{\mathrm{Jym} \mathrm{s}^{-1}}\right)$,

where $F_{\mathrm{CO}}^{\prime}$ is the velocity-integrated flux, $\nu_{\text {rest }}$ is the rest frequency of the line in $\mathrm{GHz}[230.538 \mathrm{GHz}$ for $\mathrm{CO}(2-$ $1)$ ], $z$ is the redshift, and $D_{L}$ is the luminosity distance, which we fix to $22 \mathrm{Mpc}$ (Schweizer et al. 2008). The CO luminosity is related to the molecular gas mass by

$$
M_{\mathrm{H}_{2}}=\alpha_{\mathrm{CO}} L_{\mathrm{CO}}
$$




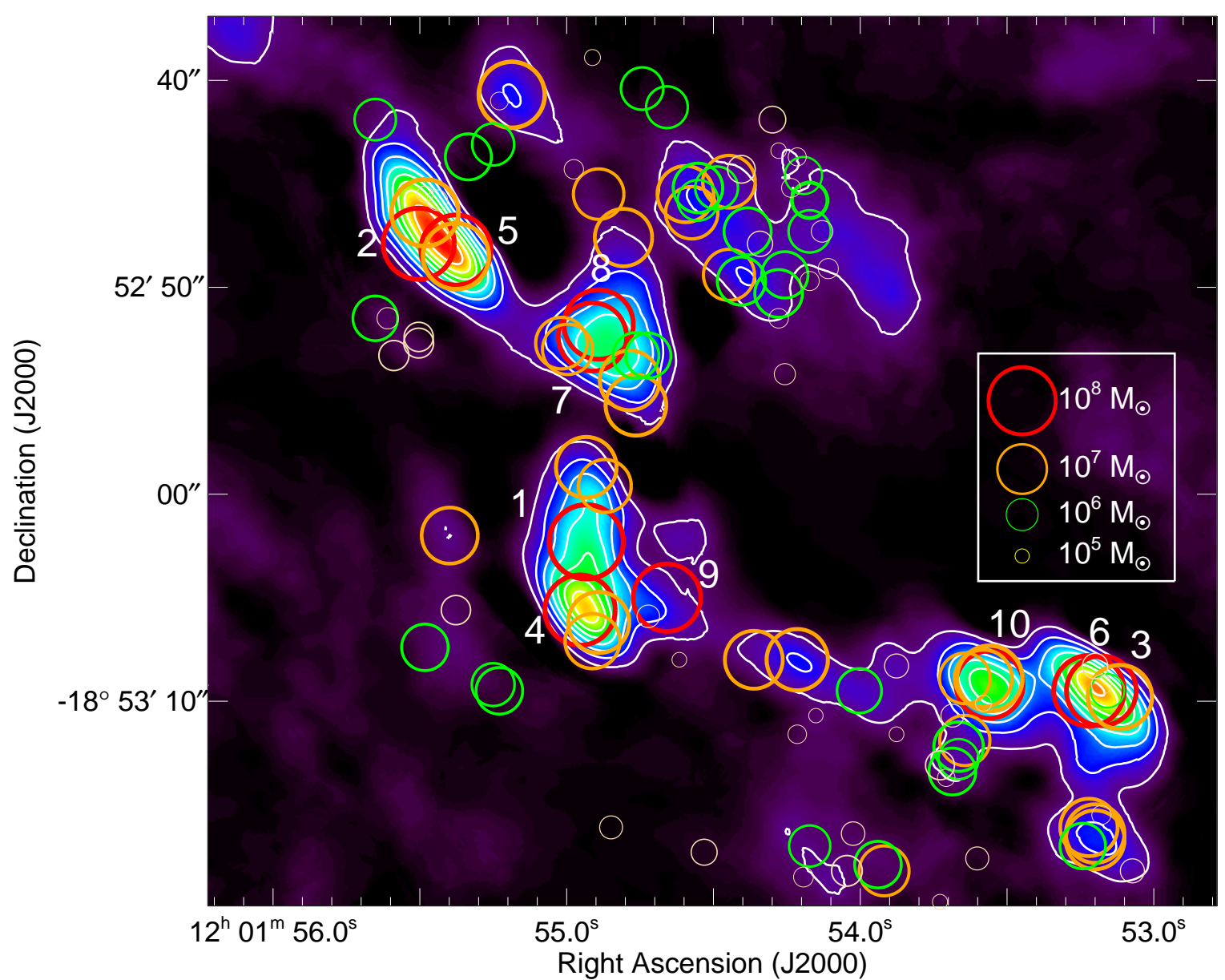

FIG. 2.-: Velocity-integrated $\mathrm{CO}(2-1)$ emission from the combined SMA+PdBI data of the overlap region of the Antennae. Contours start at 3 times the rms of the image $\left(\sigma=3.71 \mathrm{Jy}_{\text {beam }}^{-1} \mathrm{~km} \mathrm{~s}^{-1}\right)$ and increase by $3 \sigma$. Intensities range from $0-100$ Jy beam ${ }^{-1} \mathrm{~km} \mathrm{~s}^{-1}$. The synthesized beam of this image is $3^{\prime \prime} .3 \times 1^{\prime \prime} .5$, corresponding to a physical scale of $374 \times 160 \mathrm{pc}$. We denote the locations of the clouds found by clumpfind with circles. Symbol size, thickness, and color reflect the molecular gas mass of the cloud estimated from the CO brightness. We find multiple overlapping clouds, with the most massive clouds concentrated at the peaks of integrated $\mathrm{CO}$ emission and less massive clouds in the surrounding regions. Numbers indicate the clouds whose intensity-weighted velocity is shown in Figure 5. We discuss this figure in further detail in $\S 3$.

where $\alpha_{\mathrm{CO}}=2 m_{\mathrm{H}} X_{\mathrm{CO}}$.

There has been some debate over the value of $X_{\mathrm{CO}}$ in the Antennae. Zhu et al. (2003) find $X_{\mathrm{CO}}$ to be 5 13 times smaller than the Galactic value $\left(X_{\mathrm{CO}, \text { gal }}=\right.$ $\left.2.8 \times 10^{20}\left(\mathrm{~K} \mathrm{~km} \mathrm{~s}^{-1}\right)^{-1}\right)$. Analysis of resolved $\mathrm{CO}(1-0)$ clouds by Wilson et al. (2003), however, suggest that $X_{\mathrm{CO}}$ in the Antennae is much larger, with an average value of $1.3 \times 10^{20}\left(\mathrm{~K} \mathrm{~km} \mathrm{~s}^{-1}\right)^{-1}$ and within the errors of the Galactic value. More recently, models of $\mathrm{CO}$ and [C II] data by Schulz et al. (2007) suggest that $X_{\mathrm{CO}}$ does not deviate much from the Galactic value. Based on these results and for consistency with Wilson et al. (2003), we adopt $\alpha_{\mathrm{CO}}=4.8 M_{\odot}\left(\mathrm{K} \mathrm{km} \mathrm{s}^{-1} \mathrm{pc}^{2}\right)^{-1}$, which corresponds to a CO-to- $\mathrm{H}_{2}$ conversion factor $X_{\mathrm{CO}}=$ $3 \times 10^{20}\left(\mathrm{~K} \mathrm{~km} \mathrm{~s}^{-1}\right)^{-1}$.

We also assume a line intensity ratio of $I_{\mathrm{CO}(2-1)} / I_{\mathrm{CO}(1-0)}=1.0$. Zhu et al. (2003) find the $I_{\mathrm{CO}(2-1)} / I_{\mathrm{CO}(1-0)}$ line intensity ratio to range from 1.0 to 1.2 in the overlap region of the Antennae with their single-dish observations. This is also the typical value found for nearby disk galaxies, as well as the central region of the Milky Way (Braine et al.
1993; Sawada et al. 2001). We multiply $M_{\mathrm{H}_{2}}$ by 1.36 to account for heavier elements to obtain the total molecular gas mass, $M_{\text {mol }}$.

One concern, especially with the higher resolution imaging, is the problem of missing flux from the lack of short spacings in interferometric observations. In order to estimate the percentage of the total flux captured by our interferometric observations, we first estimate the total flux in the region covered by other observations. $\mathrm{CO}(1-0)$ single-dish mapping of the Antennae with the NRAO $12 \mathrm{~m}$ by Gao et al. (2001) gives a total flux of $3172 \mathrm{Jy} \mathrm{km} \mathrm{s}^{-1}$. Note that the region mapped by Gao et al. (2001) is much larger than the region covered by our observations or that of Wilson et al. (2003). They used the single-dish spectra of Gao et al. (2001) to estimate a total $\mathrm{CO}(1-0)$ flux of $1654 \mathrm{Jy} \mathrm{km} \mathrm{s}^{-1}$ in their smaller area mapped by the OVRO interferometers. We can use the estimate of Wilson et al. (2003) to determine the total $\mathrm{CO}(1-0)$ flux in the area mapped by our observations. Since our observations do not include NGC 4038, we subtract $256 \mathrm{Jy} \mathrm{km} \mathrm{s}^{-1}$ from Wilson's estimate to obtain a total single-dish $\mathrm{CO}(1-0)$ flux for our region of 
$1398 \mathrm{Jy} \mathrm{km} \mathrm{s}^{-1}$.

We can compare the predicted total CO flux with the flux measured in our interferometric observations. We determine the flux by summing up the fluxes of the clouds found by clumpfind in our combined SMA+PdBI data. We obtain a total flux of $1358 \mathrm{Jy} \mathrm{km} \mathrm{s}^{-1}$, which is very close to the single-dish flux assuming a line intensity ratio of $I_{\mathrm{CO}(2-1)} / I_{\mathrm{CO}(1-0)}=1.0$. If we assume a line intensity ratio of $I_{\mathrm{CO}(2-1)} / I_{\mathrm{CO}(1-0)}=1.1$ (Zhu et al. 2003), then we are missing only $\sim 12 \%$ of the total flux. This indicates that most of the $\mathrm{CO}$ mass may be in discrete clouds rather than distributed in the diffuse ISM in the overlap region. Note that this may not be the case for regular star-forming disks, where the gas tends to be more diffuse. Lower cutoff thresholds in clumpfind yield total fluxes of 1472 and $1614 \mathrm{Jy} \mathrm{km} \mathrm{s}^{-1}$ for the 2.5 and $2 \sigma_{\text {chan }}$ thresholds, respectively, which imply that noise clumps may be picked up as real clouds at these threshold levels.

\subsection{Basic Cloud Properties: Two Populations}

With the clumpfind parameters adopted in $\S 3.1$, we detect a total of 132 clouds. We list all the clouds and their basic properties in Table 2. As expected, if we use the lower detection thresholds, clumpfind extracts a larger number of clouds (total of 332 and 224 clouds for the 2 and $2.5 \sigma_{\text {chan }}$ thresholds, respectively). Most of the new clouds detected with the lower thresholds are low mass and unresolved - the number of clouds with $\log M_{\mathrm{mol}} / M_{\odot}<6.5$ doubles and triples with the 2.5 and $2 \sigma_{\text {chan }}$ thresholds and therefore are not significant in our analysis.

We find a large range of values for the basic properties of the 132 clouds (Figure 3 and Table 3). The molecular masses (discussed in $\S 3.2$ ) span over 3 orders of magnitude in dynamic range, from $M_{\mathrm{mol}} \approx 10^{5}$ to $2.4 \times 10^{8} M_{\odot}$, with a mean of $2.0 \times 10^{7} M_{\odot}$, a median of $3.0 \times 10^{6} M_{\odot}$, and standard deviation (calculated in $\log$ space) of 1.0 dex. One of the most interesting characteristics of the mass distribution is that it appears distinctly bimodal, with a maximum around our sensitivity limit of $M_{\mathrm{mol}} \approx 10^{5} M_{\odot}$ and clear secondary maximum near $M_{\text {mol }} \approx 10^{7} M_{\odot}$. The exact location of the minimum is somewhat arbitrary, within the range of $\log M_{\mathrm{mol}} / M_{\odot} \approx 6.3-6.7$. From considerations of the mass function ( $\S 3.7$ ), we pick a fiducial value of $\log M_{\mathrm{mol}} / M_{\odot}=6.5$ to separate the two populations. With this choice, there are 68 clouds in the low-mass group and 64 clouds in the high-mass group, whose respective median masses are $M_{\mathrm{mol}}=2.3 \times 10^{5} M_{\odot}$ and $M_{\text {mol }}=1.4 \times 10^{7} M_{\odot}$.

Values of radius and velocity dispersion estimated by clumpfind are corrected for spatial and spectral resolution following equation A7 from Williams et al. (1994). We find that the radii of the clouds span a very large range, from $r=35$ to $317 \mathrm{pc}$. Of the 132 clouds found, 64 are unresolved spatially. We assign an upper limit of $80 \mathrm{pc}$ to the radius of these clouds, half of our minimum resolution diameter of $160 \mathrm{pc}$. Using the KaplanMeier product-limit estimator (Feigelson \& Nelson 1985), which properly accounts for the upper limits, we find a mean radius of $119.2 \pm 7.3 \mathrm{pc}$ and a median value of $77.0 \mathrm{pc}$ for the entire sample. The distribution of sizes, like that of mass, appears bimodal. In fact, given the large fraction of upper limits at the small- $r$ end of the distribution, the true separation between the two peaks must be even greater than it appears. Splitting the sample into the low-mass and high-mass groups defined above, the respective mean and median radii become $r=$ $(57.0 \pm 7.1,48.0) \mathrm{pc}$ and $(180.6 \pm 8.8,170.0) \mathrm{pc}$. In light of the large number of upper limits, the statistics for the low-mass subsample should be treated with caution.

The clouds on the upper end of the size distribution are larger than any known GMCs in the Milky Way (Solomon et al. 1987; Heyer et al. 2009) or in nearby disk galaxies (Bolatto et al. 2008). While blending may be an issue (but see arguments below as to why we do not think this is a major problem), at face value the largest clouds represent a distinct class. Wilson et al. (2000) described these very large clouds as super-giant molecular complexes. Our spatial resolution of $80 \mathrm{pc}$ is high enough to resolve the most massive clouds into smaller clouds, but nonetheless they appear to be single entities. There is very good correspondence between cloud size and cloud mass; the most massive clouds are also the largest in physical extent. The corollary implication, therefore, is that the most massive clouds in our sample are intrinsically as massive as they appear. Their exceptionally high masses are not a spurious artifact of insufficient resolution. This is one of the central results of this study.

Of the 132 clouds found, 86 are resolved in velocity, with velocity dispersions $\left(\sigma_{v}\right)$ ranging from 0.8 $25.6 \mathrm{~km} \mathrm{~s}^{-1}$. For the unresolved clouds, we assign an upper limit of $4.9 \mathrm{~km} \mathrm{~s}^{-1}$, the channel size of our data cube. There is an overlap of 45 clouds that have limits in both radius and velocity dispersion. Note that a few clouds have radius and/or $\sigma_{v}$ smaller than the upper limit because they were bright enough to be resolved. Unlike the sizes and masses, the distribution of velocity dispersions is not as cleanly separated between the high-mass and low-mass groups, although lower mass clouds tend to have lower values of $\sigma_{v}$. We calculate a mean and median velocity dispersion of $7.8 \pm 0.6 \mathrm{~km} \mathrm{~s}^{-1}$ and $6.2 \mathrm{~km} \mathrm{~s}^{-1}$, respectively, for the sample as a whole. Considered separately, the low-mass and high-mass groups have mean and median dispersions of $\sigma_{v}=(2.6 \pm 0.3,2.0) \mathrm{km} \mathrm{s}^{-1}$ and $(13.3 \pm 0.6,12.0) \mathrm{km} \mathrm{s}^{-1}$, respectively. Again, the statistics of the low-mass group may be uncertain because of the large fraction $(68 \%)$ of upper limits.

\subsection{Molecular Gas and Cloud Kinematics}

We show the intensity-weighted velocity and velocity dispersion map of our combined SMA+PdBI CO(2-1) data in Figure 4. Consistent with previous interferometric studies of molecular gas in the Antennae (Gao et al. 2001; Wilson et al. 2003; Ueda et al. 2012), we find that the kinematics of the molecular gas in the overlap region are extremely complicated. Several of the $\mathrm{CO}(2-1)$ intensity peaks, especially NGC 4039 in the lower right corner, show fairly obvious velocity gradients. Intensity-weighted velocity dispersions are large - often $>50 \mathrm{~km} \mathrm{~s}^{-1}$ - most likely due to multiple clouds along the line of sight, which we will discuss later. This does not contradict our conclusion that we can resolve individual clouds because clumpfind differentiates by velocity as well as spatially.

The velocity map gives the average velocity at any 

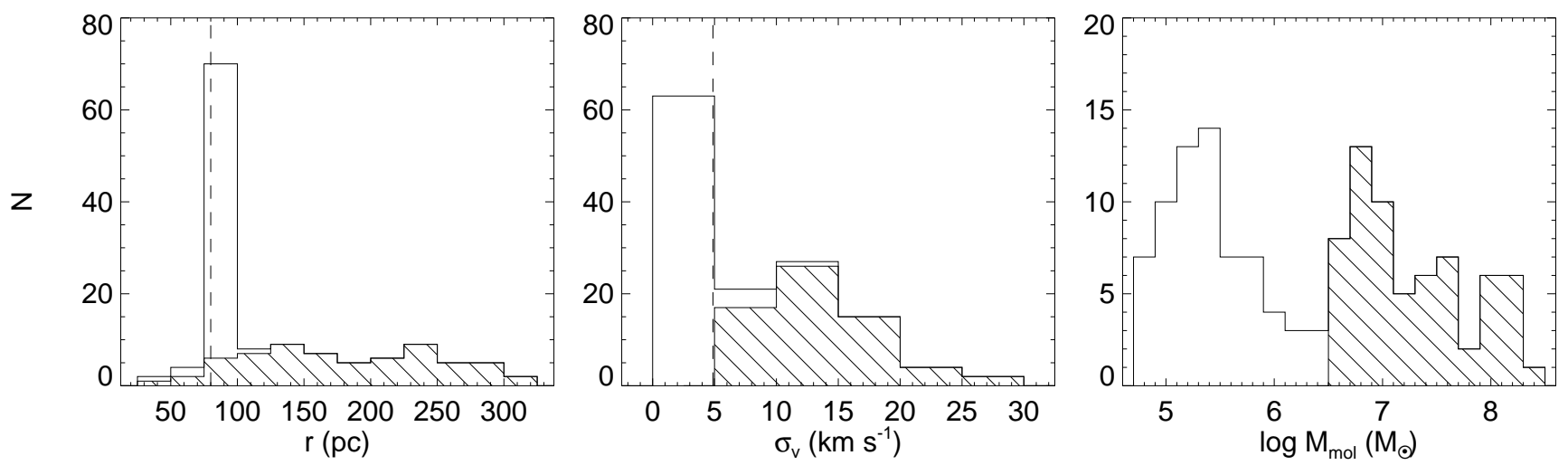

Fig. 3.- : Histogram of radius, velocity dispersion, and molecular gas mass for the clouds found by clumpfind. Clouds that were unresolved spatially or in velocity are assigned upper limits of $80 \mathrm{pc}$ and $4.9 \mathrm{~km} \mathrm{~s}^{-1}$, respectively, which we mark in the first two panels with dashed vertical lines. The clouds are broken up into two groups, above and below $\log M_{\text {mol }} / M_{\odot}=6.5$ (shaded and open histogram, respectively), which corresponds to a distinct break in the differential mass function discussed in $\S 3.7$.

TABLE 3

Cloud Statistics

\begin{tabular}{|c|c|c|c|c|c|c|c|c|c|c|c|}
\hline & Mean & $\begin{array}{c}r \\
\text { Error } \\
(\mathrm{pc})\end{array}$ & Median & Mean & $\begin{array}{c}\sigma_{v} \\
\text { Error } \\
\left(\mathrm{km} \mathrm{s}^{-1}\right)\end{array}$ & Median & Mean & $\begin{array}{c}\log M_{\mathrm{mol}} \\
\text { StDev } \\
\left(\log M_{\odot}\right)\end{array}$ & Median & $\bar{\alpha}$ & $\overline{\beta \beta}$ \\
\hline All Clouds & 119.2 & 7.3 & 77.0 & 7.8 & 0.6 & 6.2 & 7.3 & 1.0 & 6.5 & & \\
\hline $\log M_{\mathrm{mol}} / M_{\odot} \geq 6.5$ & 180.6 & 8.8 & 170.0 & 13.3 & 0.6 & 12.0 & 7.6 & 0.5 & 7.2 & $-1.44 \pm 0.14$ & $4.01 \pm 1.01$ \\
\hline $\log M_{\mathrm{mol}} / M_{\odot}<6.5$ & 57.0 & 7.1 & 48.0 & 2.6 & 0.3 & 2.0 & 5.7 & 0.4 & 5.4 & $-1.39 \pm 0.10$ & $3.02 \pm 0.59$ \\
\hline
\end{tabular}

Note. - Basic statistical properties (radius, velocity dispersion, and molecular gas mass) for the clouds extracted by clumpfind. We break up the cloud statistics into two groups, $\log M_{\mathrm{mol}} / M_{\odot} \geq 6.5$ and $\log M_{\mathrm{mol}} / M_{\odot}<6.5$, to show that there are significant differences between the general properties for these two populations. For $r$ and $\sigma_{v}$, we list the mean, the error on the mean, and the median as calculated using the Kaplan-Meier product-limit estimator to account for the upper limits. The last two columns show the results from the ordinary least squares fits to the differential mass function for the two populations (Figure 9) in log-log space, where $\log \mathrm{d} N / \mathrm{d} M_{\mathrm{mol}}=\beta+\alpha \log M_{\mathrm{mol}}$.

given position, weighted by the CO intensity, so velocity information could be blurred out if there are multiple clouds along the line of sight. To preserve some velocity information, we made individual first moment maps for the 50 brightest clouds extracted by clumpfind, using the masks provided by the algorithm. Figure 5 shows the first moment velocity maps and spectra for the 10 most massive clouds found by clumpfind in our combined SMA+PdBI data, with their spatial locations labeled in Figure 2. We find that about half of the clouds show a velocity gradient indicating shear or rotation. Cloud 10 appears to have multiple velocity components. However, because the velocity peaks are separated by less than $2 \sigma_{\text {chan }}$, clumpfind assigns the emission to a single cloud.

\subsection{Evidence for Gravitationally Bound Clouds}

We estimate the virial mass for each cloud from the resolution-corrected radius and velocity dispersion. The virial mass is given by

$$
M_{\mathrm{vir}}=\frac{5 r \sigma_{v}^{2}}{\alpha_{g} G},
$$

where $\alpha_{g}$ is a geometric factor that depends on the density profile. For a self-gravitating cloud in virial equilibrium, we expect the density profile to scale as $r^{-2}$ (Bo- denheimer \& Sweigart 1968; Shu 1977; Keto \& Caselli 2010) and $\alpha_{g}=5 / 3$. We plot the relationship between molecular gas mass and virial mass in Figure 6 . The detections alone give $\left\langle M_{\mathrm{vir}} / M_{\mathrm{mol}}\right\rangle=1.2$, but this ratio drops to $\left\langle M_{\mathrm{vir}} / M_{\mathrm{mol}}\right\rangle=1.03$ if we properly take the upper limits into account.

As mentioned in $\S 3.2$, there is some debate over whether $X_{\mathrm{CO}}$ is much smaller in the Antennae (Zhu et al. 2003; Wilson et al. 2003; Schulz et al. 2007). We show in the upper left corner of Figure 6 the distance each point would move to the left if $X_{\mathrm{CO}}$ was 2 and 5 times smaller, and to the right if $X_{\mathrm{CO}}$ was 2 times larger than our assumed value of $X_{\mathrm{CO}}=3 \times 10^{20}\left(\mathrm{~K} \mathrm{~km} \mathrm{~s}^{-1}\right)^{-1}$. Considering the many uncertainties inherent in the determination of either $M_{\mathrm{vir}}$ or $M_{\mathrm{mol}}$, the data are entirely consistent with $M_{\mathrm{vir}} / M_{\mathrm{mol}} \approx 1$. In other words, the clouds are gravitationally bound structures. This is a key point. It implies that the extreme properties of the largest clouds - their large sizes, internal velocities, and masses - are unlikely to be artifacts of spatial or velocity blending. If they were, then it would be a coincidence that the radii and velocity dispersions of the clouds would conspire to have just the right combination to produce $M_{\text {vir }} \approx M_{\text {mol }}$.

If we run clumpfind with lower thresholds, we still find a one-to-one correspondence between molecular gas and virial mass, but some of the low-mass clouds fall quite a bit below this relation. These clouds typically have $\sigma_{\mathrm{v}}<$ 

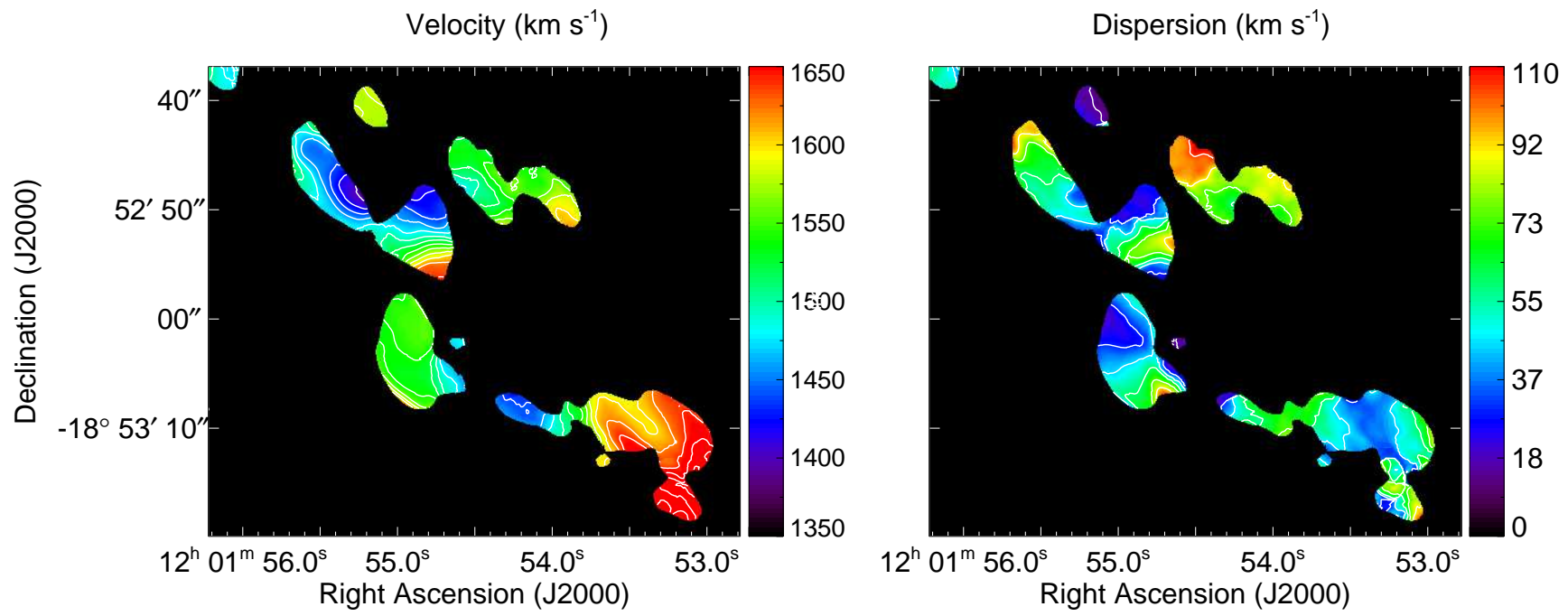

FIG. 4.- : Intensity-weighted velocity (left) and velocity dispersion (right) maps from our combined $\mathrm{SMA}+\mathrm{PdBI} \mathrm{CO}(2-1)$ data, masked at the $3 \sigma$ level of CO intensity. White contours demarcate velocities and velocity dispersions in steps of 20 and $15 \mathrm{~km} \mathrm{~s}^{-1}$, respectively.

$2 \mathrm{kms}^{-1}$ and upper limits on radius. There are only one or two clouds found using the $3 \sigma_{\text {chan }}$ threshold with the same behavior, which reinforces our earlier conclusion that some of the additional low-mass clouds extracted using the 2 and $2.5 \sigma_{\text {chan }}$ detection thresholds may not be significant above the noise.

\subsection{Cloud Scaling Relations}

Larson (1981) described the relationship between the size, linewidth (velocity dispersion), mass, and density of molecular clouds. Here we examine whether our clouds fall on these scaling relations and how they compare to typical GMCs from non-starburst galaxies.

We plot the relationship between radius $(r)$ and velocity dispersion $\left(\sigma_{v}\right)$ for our clouds in Figure 7. Solomon et al. (1987) found a relationship of the form $\sigma_{v} \propto r^{0.5}$ for Milky Way GMCs. This correlation has been shown to hold even down to very small scales within individual GMCs (Heyer et al. 2009). We find that most of our clouds fall on the $r-\sigma_{v}$ relation, extending it to larger sizes and higher velocity dispersions. However, the largest clouds in our study appear to have systematically higher velocity dispersions than predicted for Milky Way GMCs. The clouds in the Antennae with $r \gtrsim 100 \mathrm{pc}$ have velocity dispersions higher by a factor of $\sim 2$ compared to Galactic GMCs. Clouds found by Ueda et al. (2012) in their CO(3-2) observations of the Antennae, which were also obtained with the SMA and at a resolution comparable to ours, also lie above the Galactic $r-\sigma_{v}$ relation. In fact, the $\mathrm{CO}(3-2)$ dispersions are even larger than those measured in $\mathrm{CO}(2-1)$. For the 35 clouds in common between our sample and that of Ueda et al., $\left\langle\sigma_{v}(3-2) / \sigma_{v}(2-1)\right\rangle \approx 2$. Matching our $\mathrm{CO}(2-1)$ clouds with the $\mathrm{CO}(1-0)$ clouds from Wilson et al. (2003) is difficult given the difference in resolution $\left(3{ }^{\prime \prime} .2 \times 4^{\prime \prime} .9 \mathrm{com}-\right.$ pared to $3^{\prime \prime} .3 \times 1$.' 5$)$, but we note that most of the $\mathrm{CO}(1-$ 0 ) clouds have velocity dispersions $<10 \mathrm{~km} \mathrm{~s}^{-1}$, significantly smaller than the velocity dispersion observed in the $\mathrm{CO}(3-2)$ and $\mathrm{CO}(2-1)$ clouds.

The large offset in the Ueda et al. velocity dispersions are especially curious. The typical size-linewidth relation in Galactic (Larson 1981; Solomon et al. 1987) and extragalactic (Bolatto et al. 2008) clouds predicts that clouds found by higher density tracers such as $\mathrm{CO}(3-2)$ should have smaller velocity dispersions if they trace smaller size scales, precisely the opposite of what Ueda et al. (2012) observe.

There are several possible explanations for the offset in velocity dispersion for the largest clouds. Assuming that the Milky Way GMCs represented by the solid line in Figure 7 are in virial equilibrium $\left(M_{\mathrm{mol}} \approx M_{\mathrm{vir}} \propto r \sigma_{v}^{2}\right)$, the size-linewidth relation $\left(\sigma_{v} \propto r^{0.5}\right)$ found by Solomon et al. (1987) implies that $M \propto r^{2}$ — that all the Milky Way clouds have similar gas surface densities. For the GMCs in the Solomon et al. sample, this corresponds to a gas surface density $\Sigma=170 M_{\odot} \mathrm{pc}^{-2}$. If the Antennae clouds are in virial equilibrium, as they appear to be (Figure 6), then one possibility is that they have higher surface densities than Milky Way GMCs. Gao et al. (2001) find that the overlap region has exceptionally high $\mathrm{HCN}$ (a high gas density tracer, $\geq 10^{4} \mathrm{~cm}^{-3}$ ) emission, equivalent to half of the total $\mathrm{HCN}$ emission in the system. Furthermore, the overlap region is extremely bright in the far-infrared (FIR; Klaas et al. 2010), which is known to be well-correlated with HCN (Gao \& Solomon 2004). This correlation has been shown to hold for a wide range of size scales, from dense Galactic cores to extreme, highredshift starbursts (Gao \& Solomon 2004; Wu et al. 2005; Gao et al. 2007).

We can roughly estimate the average $\mathrm{H}_{2}$ number density in each cloud from its CO brightness, assuming the mass is distributed uniformly within a sphere. Larson (1981) found that the average number density $\left(\left\langle n\left(\mathrm{H}_{2}\right)\right\rangle\right)$ varies inversely as the cloud diameter $L\left[\left\langle n\left(\mathrm{H}_{2}\right)\right\rangle\left(\mathrm{cm}^{3}\right) \propto\right.$ $L(\mathrm{pc})^{-1.1}$, implying that the column density is essentially independent of size. Figure 8 shows that our clouds follow this relationship. At a given size, however, our clouds appear to be offset toward higher number densities (by $\sim 0.3$ dex) than the molecular clouds from Larson (1981), as well as GMCs from Bolatto et al. (2008). 


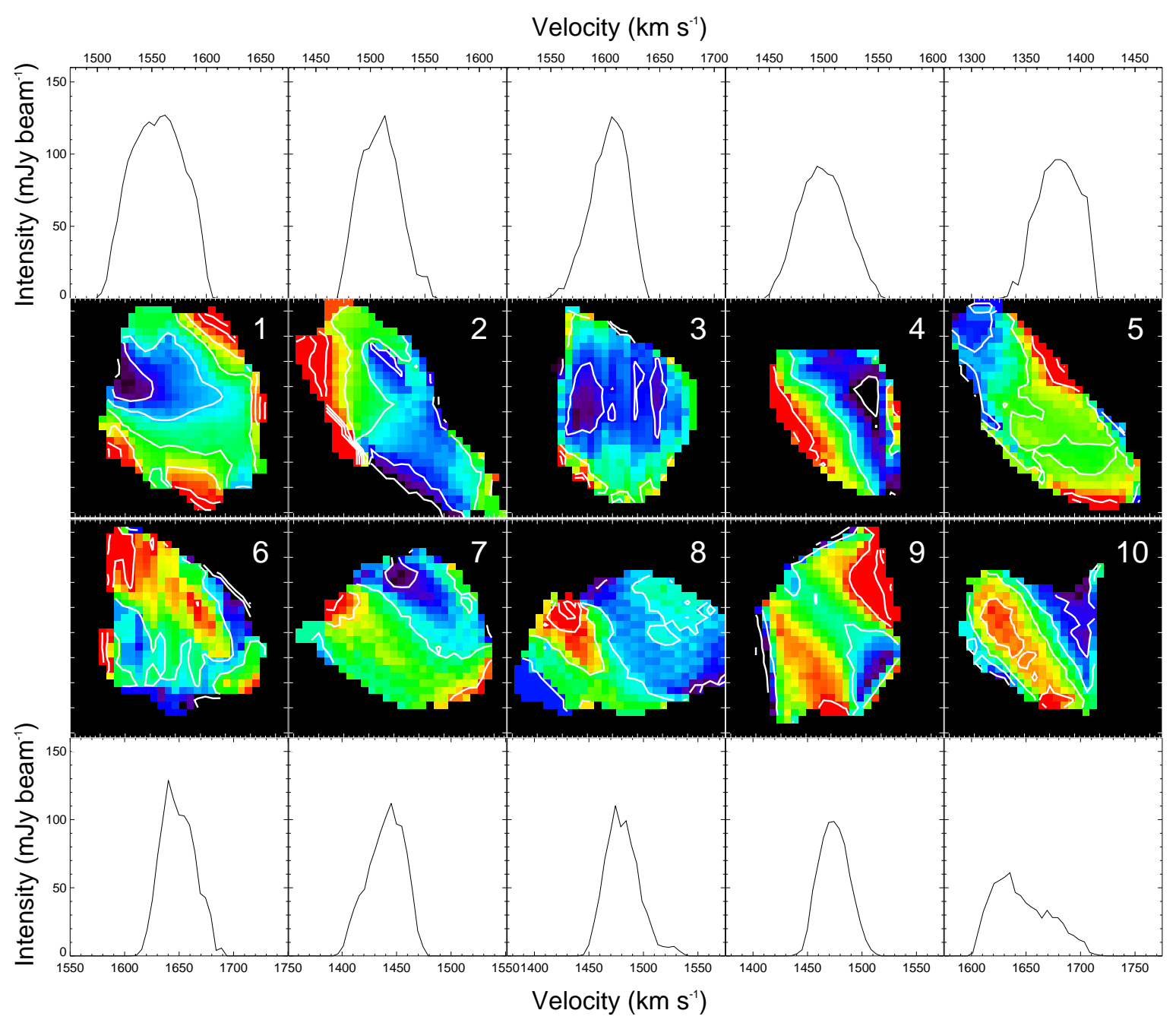

FIG. 5.- : Intensity-weighted velocity maps (middle panels) of the 10 most massive clouds extracted by clumpfind (labeled in Figure 2), showing velocity gradients indicative of shear or rotation in many of the clouds. Clouds are numbered according to decreasing CO intensity. The angular size of each panel is the same $\left(8.3^{\prime \prime} \times 8.3^{\prime \prime}\right)$, but note that the color-scale is different for each panel, chosen to best show the velocity gradient. The white contours demarcate velocities in steps of $10 \mathrm{~km} \mathrm{~s}{ }^{-1}$ for all panels. The CO spectrum of each cloud is plotted in the top/bottom panels, with channel widths of $4.9 \mathrm{~km} \mathrm{~s}^{-1}$.

This is consistent with the argument for higher surface densities, and could also be the explanation for the even larger offset of the Ueda et al. (2012) data.

Another possibility is the presence of elevated external pressure. In the Galactic center, Oka et al. (1998, 2001) find GMCs that have extremely large velocity dispersions - approximately a factor of 5 larger at a given radius than the clouds in the Galactic disk. Oka et al. (1998) argue that the large velocity dispersions from turbulence keep the clouds in equilibrium with high external pressures that arise from hot gas and/or magnetic fields in this region. The overlap region of the Antennae may also be experiencing higher pressures as the region of contact between two gas-rich galaxies undergoing a major merger. The shocks produced under such violent conditions, especially in the overlap region, could generate high interstellar pressures and high turbulence. Higher $J$ transitions of $\mathrm{CO}$ are more sensitive to shocked gas, and hence the higher velocity dispersions of the Ueda et al. clouds may be due to the increased sensitivity of $\mathrm{CO}(3-2)$ to collisions and turbulence.

Bolatto et al. (2008) attribute the GMCs that have higher velocity dispersions in their sample to blending of multiple clouds. This, too, is a distinct possibility in our data, given the complex geometry and kinematics of the overlap region in the Antennae. Recent work has shown that clumpfind has difficulty accurately measuring cloud parameters in crowded fields (e.g., Sheth et al. 2008; Pineda et al. 2009). A few of our spectra in Figure 5 show hints of multiple peaks that could be interpreted as incomplete deblending by clumpfind. Nevertheless, as argued in $\S 3.5$, we do not believe that blending seriously compromises our results, although it is difficult to rule out this effect entirely.

At this point, it is unclear what is the primary cause for the larger velocity dispersions - higher gas densities, elevated external pressure, collisions, turbulence, cloud blending, or a combination of these factors. Observations 


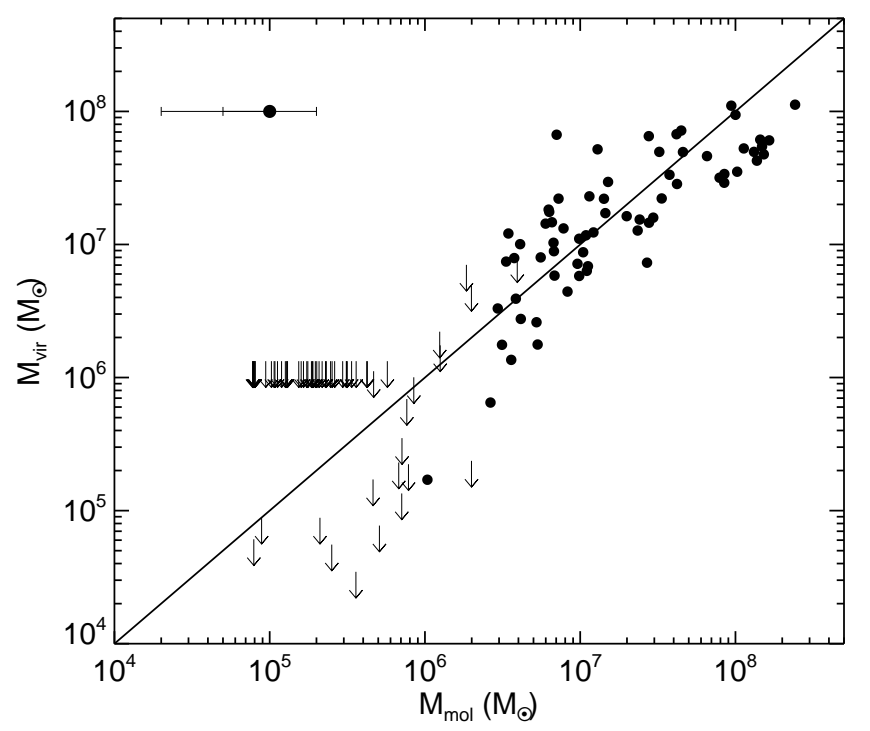

FIG. 6.- : Molecular gas mass estimated from the $\mathrm{CO}(2-$ $1)$ intensity versus the virial mass $\left(M_{\mathrm{vir}}=5 \mathrm{r} \sigma_{v}^{2} / \alpha_{g} G\right)$ estimated from the radius $r$ and velocity dispersion $\sigma_{v}$ found by clumpfind. We assume a geometrical factor $\alpha_{g}$ of $5 / 3$, which corresponds to a density profile scaling as $r^{-2}$. Arrows indicate upper limits in radius and/or velocity. We show in the upper left corner the distance each point would move to the left if $X_{\mathrm{CO}}$ was 2 and 5 times smaller, and to the right if $X_{\mathrm{CO}}$ was 2 times larger than our assumed value of $X_{\mathrm{CO}}=$ $3 \times 10^{20}\left(\mathrm{~K} \mathrm{~km} \mathrm{~s}^{-1}\right)^{-1}$. Solid line demarcates the one-to-one correspondence between the molecular gas and virial mass. Most of our points fall on or below the line, suggesting that the clouds are gravitationally bound and some may be collapsing.

at much higher angular resolution, especially of optically thin $\mathrm{CO}$ species $\left(\mathrm{C}^{17} \mathrm{O}, \mathrm{C}^{18} \mathrm{O},{ }^{13} \mathrm{CO}\right)$ and other highdensity tracers (e.g., $\mathrm{HCN}, \mathrm{HCO}^{+}$) will help distinguish between these different possibilities.

\subsection{The Mass Function}

We plot the differential mass function $\left(\mathrm{d} N / \mathrm{d} M_{\mathrm{mol}}\right)$ of the clouds found in the SMA+PdBI data in Figure 9, with bins of $\log M_{\mathrm{mol}} / M_{\odot}=0.2$. The slope of the ordinary least squares fit $(\alpha)$ corresponds to a power law of the mass function in linear space $\left(\mathrm{d} N / \mathrm{d} M_{\text {mol }} \propto\right.$ $\left.M_{\mathrm{mol}}^{\alpha}\right)$. The improved sensitivity of our observations allows us to extend the mass function down to lower masses than previous work, down to a $5 \sigma$ completeness limit of $3.8 \times 10^{5} M_{\odot}{ }^{5}$. By comparison, Wilson et al. (2003) reached a completeness limit more than 10 times higher, $5 \times 10^{6} M_{\odot}\left(6.7 \times 10^{6} M_{\odot}\right.$ if they adopted a distance of $22 \mathrm{Mpc})$.

One of the most remarkable features of the mass function is that there is a distinct break around $\log M_{\mathrm{mol}} / M_{\odot} \approx 6.3-6.7$, which coincides with the most massive Galactic clouds (Solomon et al. 1997). This break appears to be robust with respect to the detection threshold, showing up in both the 2 and $3 \sigma_{\text {chan }}$ levels. Detailed examination of the $(u, v)$ coverage and amplitude versus $(u, v)$ distance of our SMA and PdBI obser-

5 We estimate the $5 \sigma$ completeness limit following the method described in Wilson et al. (2003).

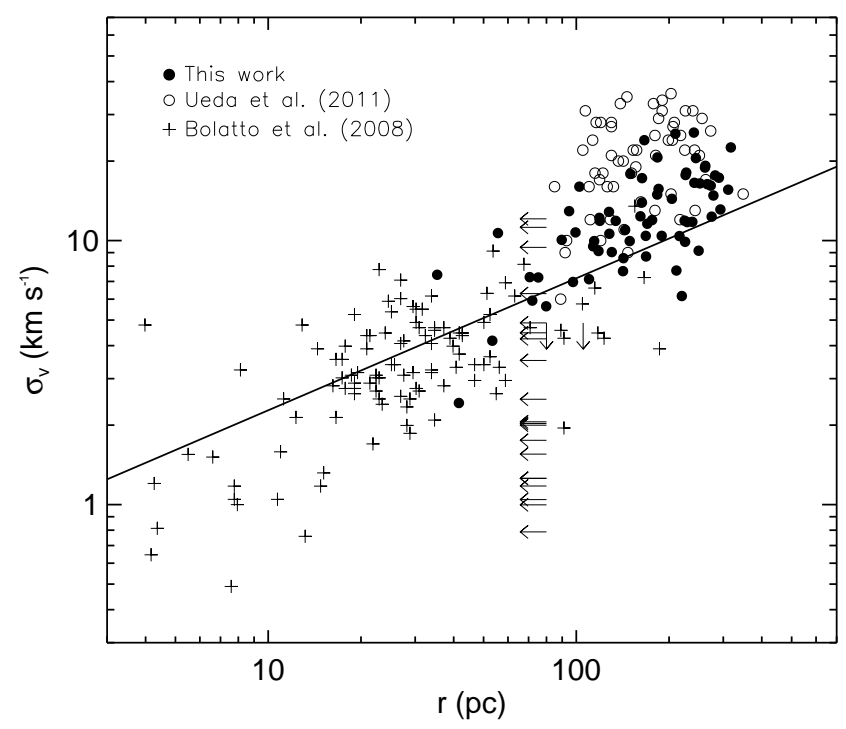

FIG. 7.- : Relationship between cloud radius $(r)$ and velocity dispersion $\left(\sigma_{v}\right)$. Clouds that were unresolved spatially or in velocity are assigned upper limits of $80 \mathrm{pc}$ (left-pointing arrow) and $4.9 \mathrm{~km} \mathrm{~s}^{-1}$ (downward arrow), respectively. Filled circles represent our data. Crosses represent GMCs from nearby galaxies (NGC 205, NGC 1569, NGC 3077, NGC 4214, NGC 4449, NGC 4605, IC 10, SMC, LMC, M33, M31) from Bolatto et al. (2008). Open circles represent clouds from the Antennae found by Ueda et al. (2012) in $\mathrm{CO}(3-2)$. The solid line demarcates the typical Milky Way GMC relationship $\sigma_{v} \approx 0.72 r^{0.5} \mathrm{~km} \mathrm{~s}^{-1}$ (Solomon et al. 1987).

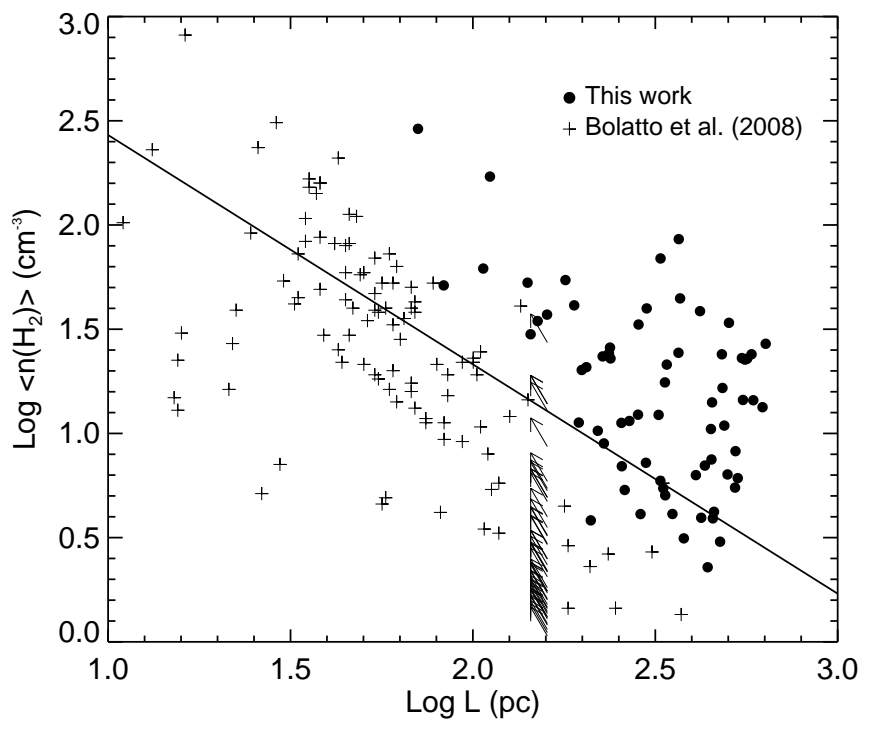

FIG. 8.-: Average $\mathrm{H}_{2}$ number density, roughly estimated as $M_{\mathrm{H}_{2}} /\left(\frac{4}{3} \pi r^{3}\right)$, as a function of cloud diameter $L$. Crosses represent extragalactic GMCs from Bolatto et al. (2008). The solid line denotes the relation $\left\langle n\left(\mathrm{H}_{2}\right)\right\rangle\left(\mathrm{cm}^{-3}\right)=$ $3400 \mathrm{~L}(p c)^{-1.1}$ found by Larson (1981). 
vations does not reveal any gaps that could introduce a break in the mass function. The break corresponds to the "gap" in the distribution of masses shown in Figure 3 and is the basis for our motivation to divide the cloud population into two subsamples.

For the sake of concreteness, we chose the threshold to be $\log M_{\mathrm{mol}} / M_{\odot}=6.5$. We perform two fits: one in the range $4.7 \leq \log M_{\mathrm{mol}} / M_{\odot}<6.5$, and a second in the range $6.5 \leq \log M_{\mathrm{mol}} / M_{\odot} \leq 8.5$. We find $\alpha=-1.39 \pm 0.10$ for the first fit and $\alpha=-1.44 \pm 0.14$ for the second fit. Figures $3 \mathrm{c}$ and 9 indicate that the mass distributions of the two populations may be log-normal, dropping off at the lower-mass end of each range. If we exclude the two end points $\left(\log M_{\mathrm{mol}} / M_{\odot}=4.8\right.$ and 6.5$)$ where the two distributions appears to turn over, the slopes for both the low- and high-mass ranges are then steeper, with $\alpha=$ $-1.52 \pm 0.08$ and $\alpha=-1.52 \pm 0.14$, respectively. These slopes are all statistically consistent with $\alpha=-1.4 \pm 0.1$ found by Wilson et al. (2003).

Note that if we use a distance of $19 \mathrm{Mpc}$ to be completely consistent with Wilson et al. (2003), the break remains apparent at $\log M_{\mathrm{mol}} / M_{\odot} \sim 6.3$ and the respective fits have slopes $\alpha=-1.64 \pm 0.16$ and $\alpha=-1.40 \pm 0.15$. Additionally, we find that if we run clumpfind with the lower detection thresholds, the slopes of the mass function at the low-mass end increases slightly (from -1.39 to -1.68), while the slope of the high-mass end remains relatively constant within the errors. We find that the break between the low- and high-mass differential mass function also remains constant with different detection thresholds; it remains $\log M_{\mathrm{mol}} / M_{\odot} \approx 6.3-6.7$ for all three thresholds that we tested.

The slopes of our mass function are well-matched to the slope of the mass function for young clusters in the Antennae, $\alpha \approx-2.0$ (Zhang \& Fall 1999; Whitmore et al. 2010). This is consistent with the good agreement between the GMC mass spectrum and the upper range of the SSC mass spectrum in M82 (Keto et al. 2005), suggesting that individual star clusters may be closely related to individual clouds.

Our fit over the mass range $6.5 \leq \log M_{\mathrm{mol}} / M_{\odot}<8.5$ suggests that some of the largest super-giant molecular complexes found by Wilson et al. break up into smaller clouds in our high-resolution observations. Importantly, many of the large clouds remain large despite the improved spatial resolution of our observations, which is sufficient to resolve the larger structures even at our highest resolution. The largest clouds in our data are much larger than GMCs in our Galaxy and typical star-forming galaxies (1-100 pc; Solomon et al. 1987; Bolatto et al. 2008).

Returning to Figure 3 and Table 3, we see that the two populations below and above the break have statistically different properties. While the clouds with $6.5 \leq \log M_{\mathrm{mol}} / M_{\odot}<8.5$ tend to be large $(r \gtrsim 100 \mathrm{pc})$ and have high velocity dispersions $\left(\sigma_{v} \gtrsim 10 \mathrm{~km} \mathrm{~s}^{-1}\right)$, the less massive clouds tend to be largely unresolved in both radius $(r \lesssim 80 \mathrm{pc})$ and velocity dispersion $\left(\sigma_{v} \lesssim 4.9\right.$ $\left.\mathrm{km} \mathrm{s}^{-1}\right)$. Also intriguing is the spatial distribution of the clouds in Figure 2 - the more massive clouds are associated with peaks of intense star formation $(\S 4)$, while the population of smaller, less massive GMCs resides in more quiescent areas far from the peaks.

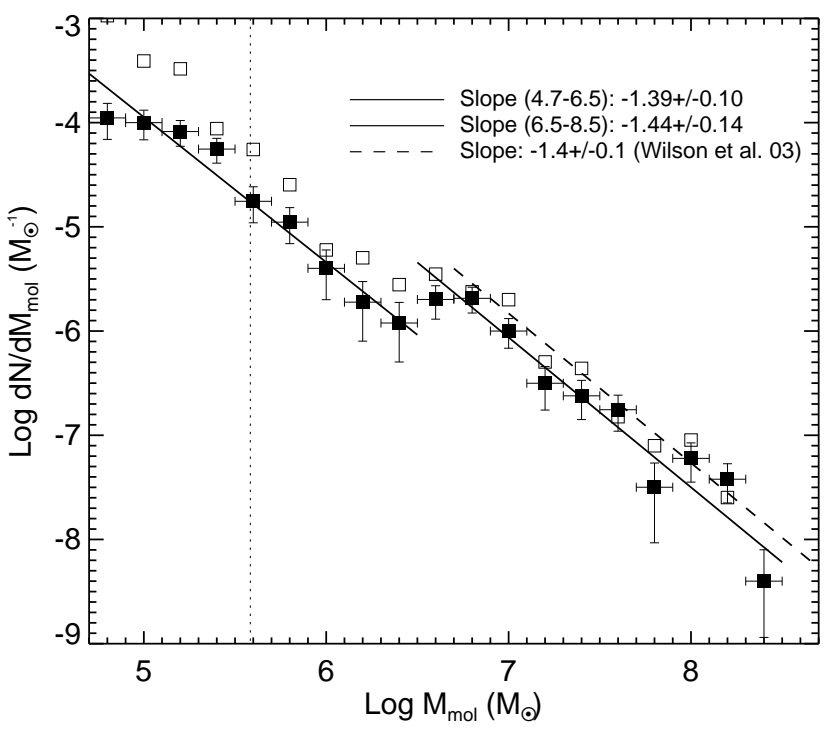

FIG. 9.-: Differential mass function for the clouds extracted by clumpfind from our combined SMA+PdBI data cube. Filled black squares show the mass function for clouds found above $3 \sigma_{\text {chan }}$, and open squares show the mass function for clouds found above $2 \sigma_{\text {chan }}$, showing that the break in the mass function is robust at both levels. Unsurprisingly, more clouds are found at the lower mass range using the $2 \sigma_{\text {chan }}$ lower level, resulting in a steeper mass function for that half of the spectrum. Horizontal error bars indicate bin sizes; vertical error bars indicate $\sqrt{(} N)$ uncertainty in each bin. The vertical dotted line demarcates the $5 \sigma$ completeness limit of our data, $3.8 \times 10^{5} M_{\odot}$. We fit two (solid) lines to the $3 \sigma_{\text {chan }}$ mass function, one for bins with $4.7 \leq \log M_{\text {mol }} / M_{\odot}<6.5$ and a second for bins with $6.5 \leq \log \bar{M}_{\text {mol }} / M_{\odot} \leq 8.5$. Both fits have slopes that are consistent with that found by Wilson et al. (2003) (dashed line).

In summary, these results suggest a bimodal distribution of giant molecular clouds/complexes in the Antennae: a regular, quiescent population that is similar to typical GMCs in nearby galaxies and the Milky Way, and a second population that appears much larger, more turbulent, and unusually massive. The exceptional properties of the largest clouds appear connected to the extreme conditions associated with the interaction zone of the merging galaxies. The dividing line between the two populations occurs near $M_{\text {mol }} \approx 3 \times 10^{6} M_{\odot}$, which, interestingly, coincides roughly with the upper end of molecular cloud masses seen in the Milky Way (Solomon et al. 1997).

\section{COMPARISON WITH STAR FORMATION TRACERS}

We explore the relationship between dense molecular gas and star formation by comparing the $\mathrm{CO}$ emission with various tracers of recent and on-going star formation.

\subsection{Super Star Clusters}

As discussed in the Introduction, SSCs are extremely young $(\lesssim$ few $-50 \mathrm{Myr})$, representing very recent star formation, and are quite prominent in the Antennae. Figure 10 shows again the velocity-integrated $\mathrm{CO}(2-1)$ emission, this time with the locations of various SSCs overplotted. These clusters are identified as compact sources in HST 
optical images (Whitmore \& Schweizer 1995; Whitmore et al. 1999; Zhang et al. 2001). All our CO clouds have SSCs nearby. However, the SSCs are identified in the optical and are not good tracers of embedded or current star formation.

\subsection{Current Star Formation}

We also consider the relationship between current star formation and the distribution and intensity of molecular gas, velocity gradients, and other signatures of shocks and compression in our $\mathrm{CO}$ data that may provide insight into the nature of SSC formation. We focus on three star formation tracers with the highest spatial resolution available - $\mathrm{H} \alpha$ emission from the recombination of gas ionized by young, massive $\mathrm{O}$ and B stars, $8 \mu \mathrm{m}$ emission from polycyclic aromatic hydrocarbons (PAHs) heated by star formation, and $4 \mathrm{~cm}$ radio continuum from either H II regions or young supernova remnants. The $\mathrm{H} \alpha$ data were taken with the $\mathrm{F} 658 \mathrm{~N}$ filter on the HST Wide Field Planetary Camera 2 (WFPC2) by Whitmore et al. (1999), the PAH maps were acquired using the Spitzer Infrared Array Camera (IRAC) by Wang et al. (2004), and the $4 \mathrm{~cm}$ continuum map was taken by Neff \& Ulvestad (2000) with the Very Large Array (VLA).

Wang et al. (2004) estimate that $\sim 5 \%$ of the $8 \mu \mathrm{m}$ emission comes from diffuse gas heated by older stars, so we subtract this component from the $8 \mu \mathrm{m}$ image following Pahre et al. (2004), by scaling the 3.6 and $4.5 \mu \mathrm{m}$ channels to match the colors of M0 III stars. Comparison between $4 \mathrm{~cm}$ continuum emission and $8 \mu \mathrm{m}$ PAH emission shows very good spatial correlation between the two tracers (Figure 11), so we elect to show only the $8 \mu \mathrm{m}$ $\mathrm{PAH}$ emission in the next figure. Figure 11 also notes the location of peaks in the FIR emission at $70 \mu \mathrm{m}$ (from the Herschel Photodetector Array Camera \& Spectrometer PACS; Klaas et al. 2010), showing good agreement with the $8 \mu \mathrm{m} \mathrm{PAH}$ and $4 \mathrm{~cm}$ continuum emission within the 5.5 resolution of the FIR image.

Figure 12 shows the velocity-integrated emission (zeroth moment; top), the intensity-weighted velocity (first moment; middle), and the intensity-weighted velocity dispersion (second moment; bottom) of our combined $\mathrm{SMA}+\mathrm{PdBI} \mathrm{CO}(2-1)$ data. Each panel is overlaid with contours of $\mathrm{H} \alpha$ (left) or $8 \mu \mathrm{m}$ PAH (right) emission to show the distribution of star formation.

As discussed in $\S 3$, there appear to be multiple overlapping clouds along the line of sight in the CO-bright regions of our data. This means that in a straight first moment map, much of the velocity information is lost due to averaging. Another way to view the velocity distribution of the molecular gas would be to consider positionvelocity slices of emission at each of the star-forming peaks (Figure 13). We choose the position-velocity slices to show the largest velocity gradients in the $\mathrm{CO}$ emission.

We discuss the prominent star-forming peaks in the $8 \mu \mathrm{m}$ map, labeled $\mathrm{A}-\mathrm{F}$ in the top right panel of Figure 12 , and consider the molecular gas distribution and kinematics at these peaks.

Peak A: Star formation in this region is seen both in $\mathrm{H} \alpha$ and PAH, both of which appear to be slightly offset from the peak $\mathrm{CO}$ emission. The higher resolution $\mathrm{H} \alpha$ image shows that the star formation breaks up into two or three peaks, one of which is almost completely offset from the CO. Brandl et al. (2009) estimate a SFR of
$0.74 M_{\odot} \mathrm{yr}^{-1}$ for this whole region based on its infrared luminosity. This is also one of the brighter regions in terms of integrated $\mathrm{CO}$ intensity. The position-velocity diagram in Figure 13 shows two or three clouds spatially separated by $\sim 3^{\prime \prime}(\sim 321 \mathrm{pc})$. The spread in gas velocity is very large, ranging from 1340 to $1540 \mathrm{~km} \mathrm{~s}^{-1}$, but there is only the slightest hint of a velocity gradient for the upper left CO cloud. Peak A is associated with water maser emission (Brogan et al. 2010), indicating very young star formation.

Peak B: One of the fainter star-forming peaks based on the PAH and $4 \mathrm{~cm}$ emission, this region barely shows up in the $\mathrm{H} \alpha$ imaging. The integrated $\mathrm{CO}$ emission is also one of the weakest, although there may be two clouds at around the same velocity, spatially separated by $\sim 3^{\prime \prime} .5$ $(\sim 375 \mathrm{pc})$.

Peak C: A region where the $\mathrm{H} \alpha$ emission may be mostly obscured, the $8 \mu \mathrm{m}$ PAH peak is slightly offset from the $\mathrm{CO}$ peak. This is one of the most highly obscured areas in the overlap region, with $A_{V}=10.3-11.8 \mathrm{mag}$ (Snijders et al. 2007; Mengel et al. 2005). The SFR in this region is $0.66 M_{\odot} \mathrm{yr}^{-1}$ (Brandl et al. 2009). The velocity dispersion of the CO is extremely large, $>300 \mathrm{~km} \mathrm{~s}^{-1}$, with at least two clouds with large velocity gradients, seen in both the middle panel of Figure 12 and the upper right panel of Figure 13. Near-infrared spectroscopic imaging by Herrera et al. (2011) also find extremely broad line widths in the warm $\mathrm{H}_{2}$ emission, which they attribute to shocks driven by gas dynamics on large scales. A compact, warm $\mathrm{H}_{2}$ source observed in this region is thought to be associated with a massive cloud on its way to becoming a SSC (Herrera et al. 2011).

Peak D: This is one of the brightest peaks in $8 \mu \mathrm{m}$ $\mathrm{PAH}$ and in $\mathrm{CO}(2-1)$. The large amount of dust and gas present explains why this peak is almost completely obscured in the $\mathrm{H} \alpha$ image. Brandl et al. (2009) estimate the SFR for this region to be large, almost $2 M_{\odot} \mathrm{yr}^{-1}$. This region also contains a water maser (Brogan et al. 2010). Multi-wavelength spectral energy distributions suggest that some of the youngest clusters in the Antennae reside here (Zhang et al. 2010). The position-velocity diagram shows at least two clouds ${ }^{6}$ with large velocity gradients, possibly in the process of merging. The molecular gas spans a large range in velocity, $\sim 200 \mathrm{~km} \mathrm{~s}^{-1}$, with $\sim 80 \mathrm{~km} \mathrm{~s}^{-1}$ separation between the two clouds.

Peak E: This peak has the brightest $\mathrm{H} \alpha$ and second brightest $8 \mu \mathrm{m}$ PAH emission, but it contains surprisingly small amounts of CO. Brandl et al. (2009) estimate a SFR of $1.8 M_{\odot} \mathrm{yr}^{-1}$ for this region from its infrared luminosity. Figure 10 shows that the youngest SSCs found by Whitmore et al. (1999) are clustered around this region. This suggests that peak $\mathrm{E}$ is a site of very recent and vigorous star formation, but given the lack of molecular gas present, star formation will not be able to continue at the same rate for much longer. The distribution of the remaining molecular gas is also interesting; the curved shape in the position-velocity diagram is suggestive of gas infalling toward the center.

Peak F (NGC 4039): This region contains the nucleus

\footnotetext{
6 Note that the contours of $\mathrm{CO}$ emission are scaled from the faintest cloud, so the lowest contour here is $2 \times$ brighter than the lowest contour of peak B.
} 


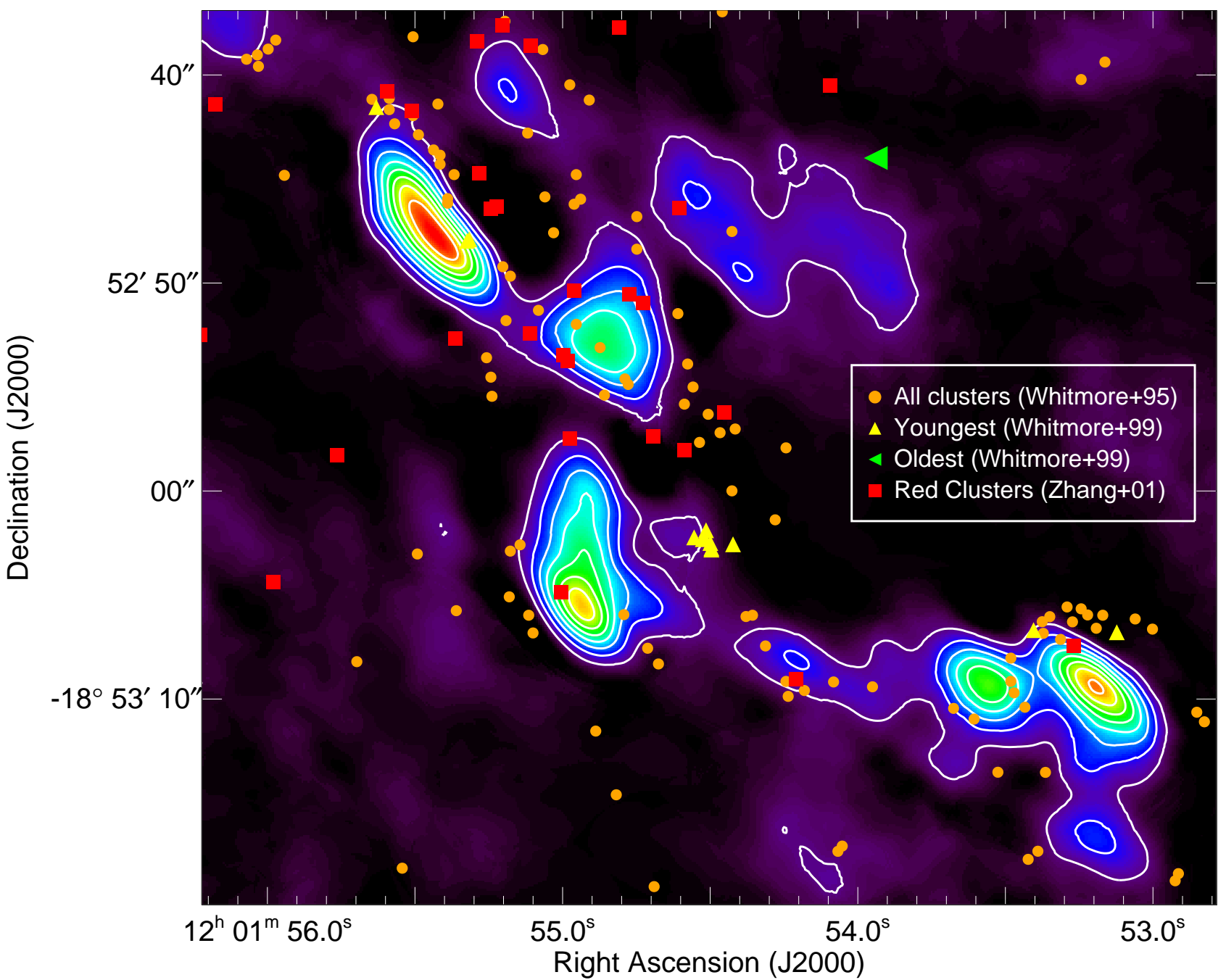

Fig. 10.- : Velocity-integrated $\mathrm{CO}(2-1)$ emission from the overlap region of the Antennae, with the same contours as Figure 2. Symbols mark the locations of SSCs of different ages identified by Whitmore \& Schweizer (1995) and Whitmore et al. (1999), as well as red clusters from Zhang et al. (2001), both of which appear to preferentially surround the CO peaks. Note that the red clusters from Zhang et al. (2001) are candidates for the youngest clusters ( $5 \mathrm{Myr}$ ), as the reddening is thought to come from dust obscuration.

of NGC 4039, one of the galaxies in the merging Antennae pair. Associated with one of the water masers found by Brogan et al. (2010), the age of star clusters in this area appears quite young, less than 6.3 Myr (Bastian et al. 2009). The SFR from the infrared luminosity, however, appears much lower than in the other peaks in the overlap region $\left(\sim 0.33 M_{\odot} \mathrm{yr}^{-1}\right.$; Brandl et al. 2009). Activity from an active galactic nucleus, if any, is extremely weak, and the infrared emission appears to be dominated by purely young star formation (Brandl et al. 2009). The distribution of the $\mathrm{H} \alpha$ emission is quite different and offset from the $8 \mu \mathrm{m}$ PAH emission. There are at least two large clouds in the $\mathrm{CO}(2-1)$ emission in this region, spanning a velocity range of $\sim 200 \mathrm{~km} \mathrm{~s}^{-1}$. One of the clouds (right) show a fairly obvious velocity gradient, while the other (left) does not. The peaks are separated spatially by $\sim 4^{\prime \prime}(\sim 428 \mathrm{pc})$.

\subsection{Implications}

Our comparison of the molecular gas distribution and kinematics with different star formation tracers $(8 \mu \mathrm{m}$ $\mathrm{PAH}, 4 \mathrm{~cm}$ continuum, and $\mathrm{H} \alpha$ ) shows that the molecular gas at star-forming peaks have large velocity dispersions, typically spanning velocities in the range of 200 $300 \mathrm{~km} \mathrm{~s}^{-1}$. In addition, we find that the molecular gas at all but two of the star-forming peaks ( $\mathrm{B}$ and $\mathrm{E}$ ) have large velocity gradients.

Keto et al. (2005) find that the clouds with active star formation in the starburst galaxy M82 are experiencing shock-driven compression, with both lateral and line-ofsight velocity gradients (i.e., inverse P Cygni profiles) within the individual clouds. These observations suggest that individual massive star clusters may have formed from individual GMCs crushed by a sudden galactic-scale increase in external pressure. While we do not observe signatures of inverse $\mathrm{P}$ Cygni profiles in our data (the presence of multiple clouds along the line of sight may blur out such signatures), the large velocity gradients we 


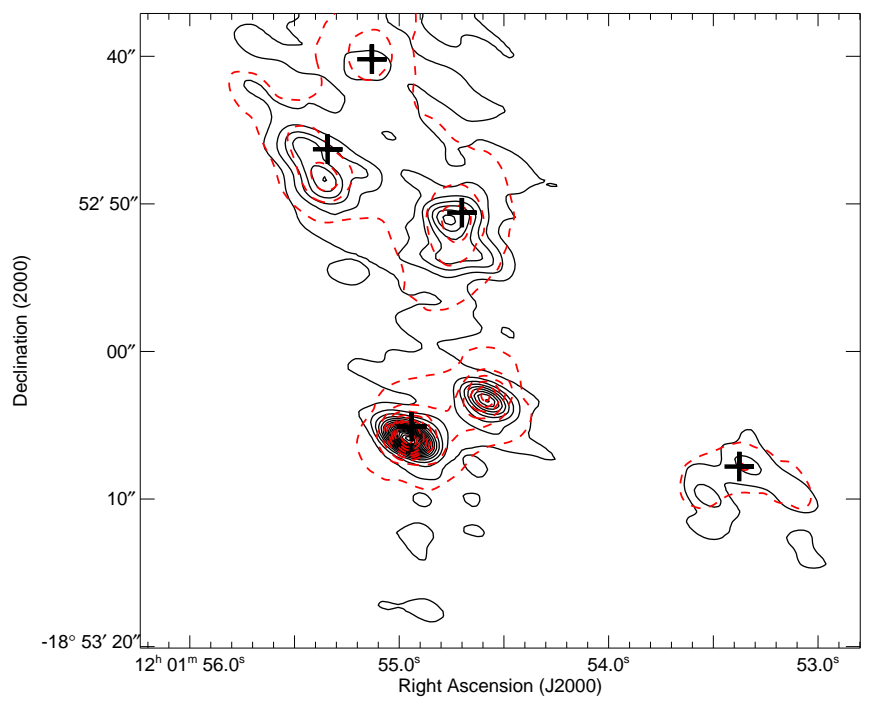

FIG. 11.-: Contours of $4 \mathrm{~cm}$ continuum (black; Neff \& Ulvestad 2000) and $8 \mu \mathrm{m}$ PAH emission (red; Wang et al. 2004) in the overlap region of the Antennae, showing very good agreement between the two. Contour levels range from 5\%$95 \%$ of peak intensity in steps of $5 \%$ for the $4 \mathrm{~cm}$ continuum emission and $10 \%-90 \%$ of peak intensity in steps of $10 \%$ for the $8 \mu \mathrm{m}$ PAH emission. FIR emission peaks from Herschel PACS $70 \mu \mathrm{m}$ imaging are marked with crosses, consistent with the $8 \mu \mathrm{m}$ PAH and continuum peaks within its 5.5 resolution limit. Note that the absence of a FIR counterpart for the central $8 \mu \mathrm{m} \mathrm{PAH} / 4 \mathrm{~cm}$ continuum peak is due to the coarser resolution of the $70 \mu \mathrm{m}$ imaging blending the two peaks together.

observe in some of the star-forming peaks may reflect compressive shocks.

Recent work provides additional observational evidence supporting the pressure-triggered formation scenario. As mentioned in the previous section, detailed examination of near-infrared emission in Peak C suggest that the warm $\mathrm{H}_{2}$ emission is excited by large-scale shocks (Herrera et al. 2011). Based on the ages of starforming regions, Zhang et al. (2010) propose two paths of shock-induced sequential star formation, perhaps propagated by the collision of GMCs, across the overlap region of the Antennae. Both paths start from the center of the overlap region (around Peak D in Figure 12) and move outwards to the north-west and south-east regions. This is well-correlated with the region with the highest star formation efficiency (Gao et al. 2001) and FIR emission (Klaas et al. 2010).

Additionally, Whitmore et al. (2010) find evidence for shock-triggered sequential cluster formation on a small scale, within the individual star-forming regions, based on the spatial distribution of clusters of different ages. One example of this sequential cluster formation highlighted by Whitmore et al. (2010) involves Peak E in our work, where an older cluster $(10-50 \mathrm{Myr})$ to the right may have triggered the formation of much younger star clusters to the left (Figure 10). And finally, simulations of the merger between NGC 4038 and NGC 4039 suggest that compressive tides occur frequently and play an important role in the evolution of the Antennae (Renaud et al. 2009).
All this clearly ties in with our results from $\S 3$. The largest clouds are associated with regions of intense infrared emission, active star formation, and high star formation efficiencies (Evans et al. 1997; Mirabel et al. 1998; Gao et al. 2001; Brandl et al. 2009; Klaas et al. 2010), where the molecular gas appear to have large velocity dispersions and gradients $(\S 4.2)$. These clouds are different than typical GMCs in nearby star-forming galaxies. While they appear to be gravitationally bound, many of these clouds are larger, more massive, and have higher velocity dispersions than expected from typical GMC scaling relations. Given that they reside in such an extreme environment, some combination of higher gas densities, elevated external pressure, turbulence, and cloud collisions is most likely responsible for the formation of these massive clouds, resulting in the bimodal distribution we observe in the cloud mass spectrum.

\section{SUMMARY}

In this paper, we present high-resolution SMA and PdBI CO(2-1) imaging of the intensely star-forming overlap region in the Antennae galaxies in order to study the properties of molecular gas that appears to be forming SSCs.

Using clumpfind, we resolve the $\mathrm{CO}(2-1)$ emission into a large number of clouds. This extends the cloud mass function down to lower masses than previous work by Wilson et al. (2003), down to a $5 \sigma$ completeness limit of $3.8 \times 10^{5} M_{\odot}$. A break in the cloud mass function around $\log M_{\mathrm{mol}} / M_{\odot} \approx 6.5$ suggests two distinct population of clouds. Fitting a broken power law to the mass function, we find that $\mathrm{dN} / \mathrm{d} M_{\mathrm{mol}} \propto M_{\mathrm{mol}}^{-1.39}$ for the lowmass cloud population and $\mathrm{dN} / \mathrm{d} M_{\mathrm{mol}} \propto M_{\mathrm{mol}}^{-1.44}$ for the high-mass cloud population, close to the $M^{-2}$ mass function found for SSCs in the Antennae. We find that the two populations have statistically different properties, and appear to populate different areas in the overlap region.

The low-mass population of clouds $\left(\log M_{\mathrm{mol}} / M_{\odot}<\right.$ $6.5)$, often below our spatial and velocity resolution limit, appears to have properties similar to GMCs in typical star-forming galaxies. These clouds tend to be distributed along the outskirts of the overlap region, away from sites of intense star formation.

The high-mass cloud population $\left(\log M_{\mathrm{mol}} / M_{\odot} \geq\right.$ 6.5 ), is associated with sites of peak star formation. These clouds appear to be much larger in size (median $r=170 \mathrm{pc}$ ) and mass (median $\log M_{\mathrm{mol}} / M_{\odot}=7.2$ ) compared to typical GMCs. While we find that these clouds appear to be in virial equilibrium $\left(M_{\mathrm{vir}} \sim M_{\mathrm{mol}}\right)$, they have higher velocity dispersions in the size-linewidth relation than expected for typical GMCs.

Comparison between our $\mathrm{CO}$ data and star formation traced by $8 \mu \mathrm{m} \mathrm{PAH} / 4 \mathrm{~cm}$ continuum $/ \mathrm{H} \alpha$ imaging show good correlation between the distribution of molecular gas and current star formation. Consistent with observational evidence of pressure-triggered star formation in the region, position-velocity slices of the $\mathrm{CO}$ data reveal large velocity gradients and dispersions in the gas at the star-forming peaks, indicative of an active, turbulent environment where compressive shocks may dominate. These results suggest that factors such as elevated external pressure, increased turbulence, and cloud collisions 

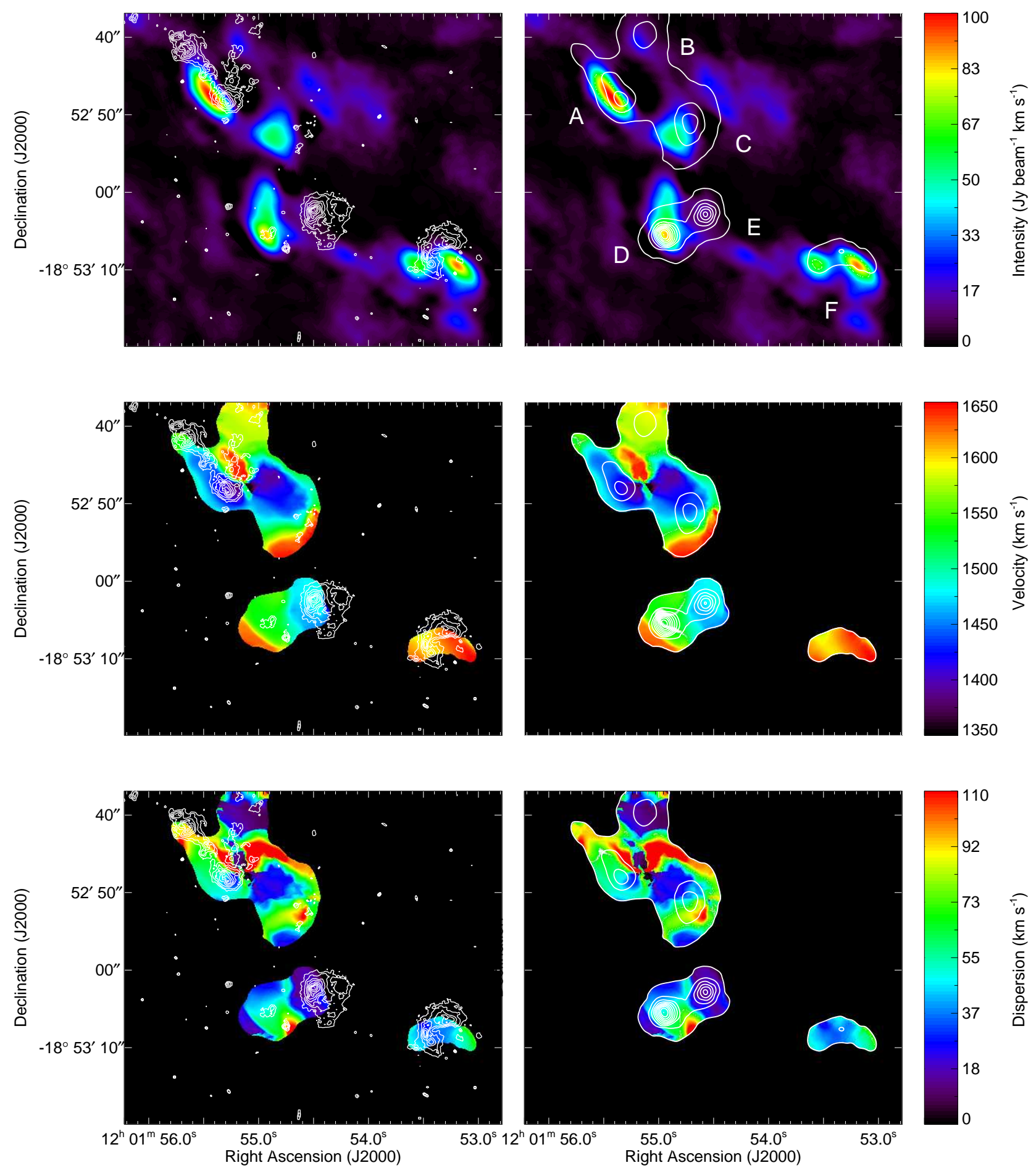

FIG. 12.-: Color-scale images of the velocity-integrated emission (zeroth moment), intensity-weighted velocity (first moment), and intensity-weighted velocity dispersion (second moment) from our combined SMA+PdBI $\mathrm{CO}(2-1)$ data from top to bottom, respectively. The first and second moments are masked below $10 \%$ of peak PAH intensity to suppress the noise in regions of weak CO emission. Color bars on the right indicate the range displayed in each panel; left and right panels are identical. The left panels are overlaid with contours of $\mathrm{H} \alpha$ emission (Whitmore et al. 1999), and the right panels are overlaid with contours of $8 \mu \mathrm{m}$ PAH emission (Wang et al. 2004), showing the distribution of star formation. Contour levels range from $0.02-1.57$ counts s $^{-1}$ in equal steps of 0.24 dex for the H $\alpha$ emission and $10 \%-90 \%$ of peak intensity in steps of $10 \%$ for the $8 \mu \mathrm{m}$ PAH emission. The star-forming peaks discussed in the text and Figure 13 are labeled A-F in the top right panel. 

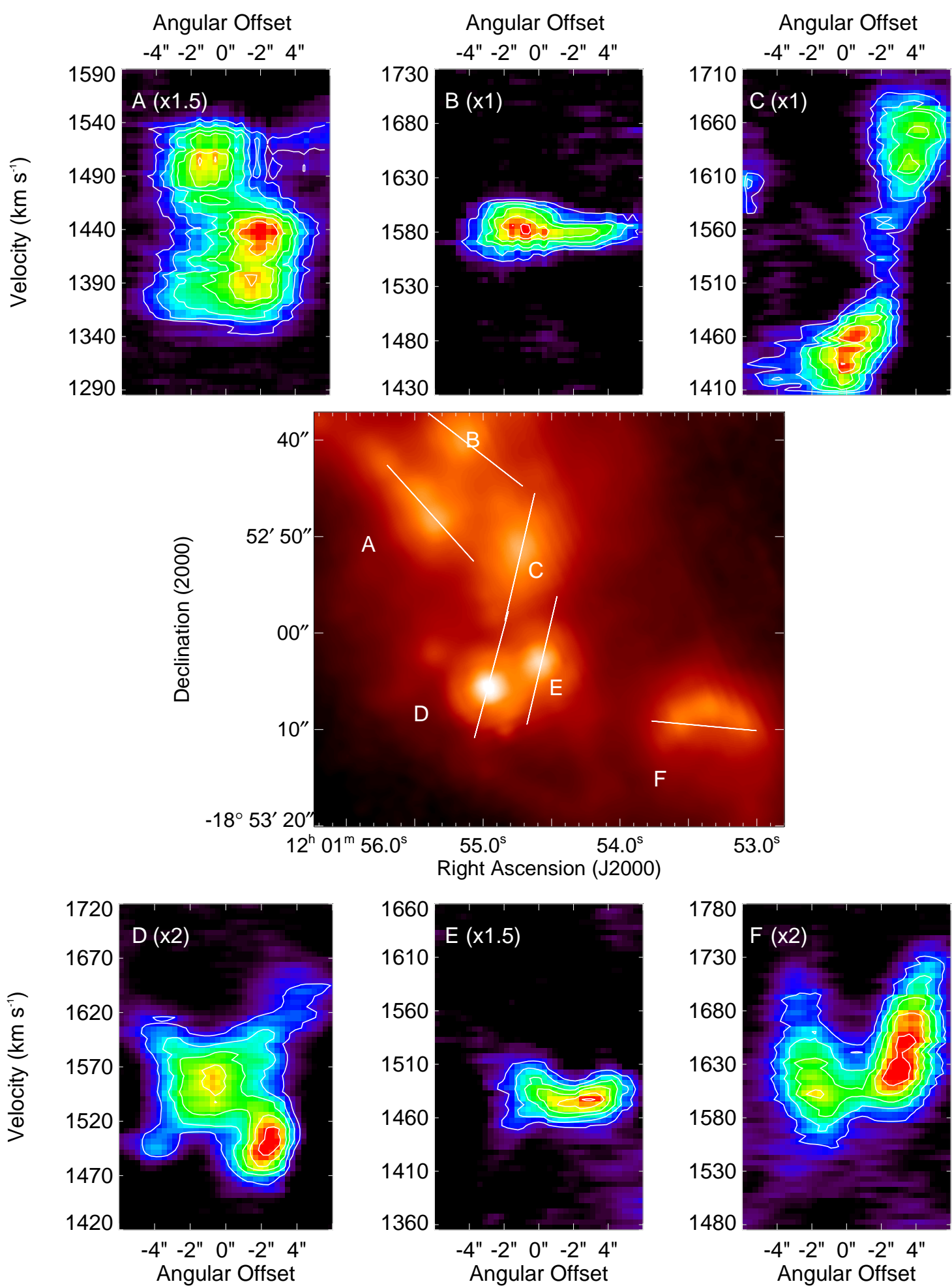

FIG. 13.- : Position-velocity diagrams of $\mathrm{CO}(2-1)$ emission (upper and lower panels) from our combined SMA+PdBI data, with cuts made at the star-forming peaks as traced by $8 \mu \mathrm{m}$ PAH emission (center panel in heat scale; the cuts are demarcated by white lines and labeled A-F to match Figure 12). Cuts are chosen to show the largest velocity gradient in the CO emission. The top and bottom of each slice corresponds to the left and right side of each panel, respectively. Contour levels are 20,35, 50, 65, 80, and 95\% of the peak intensity of the cut with the weakest emission (Panel C, $0.47 \mathrm{Jy}_{\text {beam }}{ }^{-1}$ ), multiplied by a factor noted in each panel to better display the cuts with stronger emission. Color scale of each image is also adjusted accordingly. The position-velocity diagrams show that the structure of the molecular gas is often extremely complex at the star-forming peaks, showing multiple peaks and velocities spanning up to $300 \mathrm{~km} \mathrm{~s}^{-1}$, suggesting that this is a very dynamically active region. 
are most likely responsible for the apparent high velocity dispersions and gas densities, resulting in the formation of the high-mass clouds and complexes we observe in the overlap region. These high-mass clouds and complexes may, in turn, form the SSCs that are so prevalent in the Antennae.

While our observations have allowed us to probe the properties of the molecular gas at much higher angular resolution and sensitivity than previous work, we are still limited to size scales much larger than that of typical star clusters $(<10 \mathrm{pc})$. With the advent of the full extended array with ALMA, sensitive and very highresolution $\left(<0^{\prime \prime} .1\right)$ imaging of the ISM in the Antennae will show whether the large, massive clouds we observe will begin to break down into smaller objects and steepen the cloud mass function.

We thank the anonymous referee for his/her helpful comments which improved this manuscript. We thank Zhao-Yu Li for help in generating the three-color image in Figure 1. We are grateful to Alberto Bolatto, Izaskun Jimenez-Serra, and Peter Teuben for helpful discussions. The research of L.C.H. is supported by the Carnegie Institution for Science. The Submillimeter Array is a joint project between the Smithsonian Astrophysical Observatory and the Academia Sinica Institute of Astronomy and Astrophysics and is funded by the Smithsonian Institution and the Academia Sinica. This work is based in part on archival data obtained with the Spitzer Space Telescope, which is operated by the Jet Propulsion Laboratory, California Institute of Technology under a contract with NASA.

\section{REFERENCES}

Ashman, K. M., \& Zepf, S. E. 2001, AJ, 122, 1888

Barth, A. J., Ho, L. C., Filippenko, A. V., \& Sargent, W. L. 1995 AJ, 110, 1009

Bastian, N., Trancho, G., Konstantopoulos, I. S., \& Miller, B. W. 2009, ApJ, 701, 607

Bodenheimer, P., \& Sweigart, A. 1968, ApJ, 152, 515

Bolatto, A. D., Leroy, A. K., Rosolowsky, E., Walter, F., \& Blitz, L. 2008, ApJ, 686, 948

Braine, J., Combes, F., Casoli, F., Dupraz, C., Gerin, M., Klein, U., Wielebinski, R., \& Brouillet, N. 1993, A\&AS, 97, 887

Brandl, B. R., et al. 2009, ApJ, 699, 1982

Brogan, C., Johnson, K., \& Darling, J. 2010, ApJ, 716, L51

Cen, R. 2001, ApJ, 560, 592

Elmegreen, B. G., \& Efremov, Y. N. 1997, ApJ, 480, 235

Evans, R., Harper, A., \& Helou, G. 1997, in Extragalactic Astronomy in the Infrared, ed. G. A. Mamon, T. X. Thuan, \& J. Tran Thanh Van, 143

Fabbiano, G., Zezas, A., King, A. R., Ponman, T. J., Rots, A., \& Schweizer, F. 2003, ApJ, 584, L5

Fabbiano, G., Zezas, A., \& Murray, S. S. 2001, ApJ, 554, 1035

Fall, S. M., Chandar, R., \& Whitmore, B. C. 2005, ApJ, 631, L133

Feigelson, E. D., \& Nelson, P. I. 1985, ApJ, 293, 192

Gao, Y. 2008, Nature, 452, 417

Gao, Y., Carilli, C. L., Solomon, P. M., \& Vanden Bout, P. A. 2007, ApJ, 660, L93

Gao, Y., Lo, K. Y., Lee, S.-W., \& Lee, T.-H. 2001, ApJ, 548, 172

Gao, Y., \& Solomon, P. M. 2004, ApJ, 606, 271

Harris, W. E., \& Pudritz, R. E. 1994, ApJ, 429, 177

Herrera, C. N., Boulanger, F., \& Nesvadba, N. P. H. 2011, A\&A, 534, A138

Heyer, M., Krawczyk, C., Duval, J., \& Jackson, J. M. 2009, ApJ, 699, 1092

Hibbard, J. E., van der Hulst, J. M., Barnes, J. E., \& Rich, R. M. 2001, AJ, 122, 2969

Hibbard, J. E., et al. 2005, ApJ, 619, L87

Ho, L. C. 1997, in Revista Mexicana de Astronomia y Astrofisica, vol. 27, Vol. 6, Revista Mexicana de Astronomia y Astrofisica Conference Series, ed. J. Franco, R. Terlevich, \& A. Serrano, 5

Ho, L. C., \& Filippenko, A. V. 1996a, ApJ, 466, L83

-. 1996b, ApJ, 472, 600

Ho, P. T. P., Moran, J. M., \& Lo, K. Y. 2004, ApJ, 616, L1

Holtzman, J. A., et al. 1992, AJ, 103, 691

Jog, C. J., \& Solomon, P. M. 1992, ApJ, 387, 152

Keto, E., \& Caselli, P. 2010, MNRAS, 402, 1625

Keto, E., Ho, L. C., \& Lo, K.-Y. 2005, ApJ, 635, 1062

Kilgard, R. E., Kaaret, P., Krauss, M. I., Prestwich, A. H., Raley, M. T., \& Zezas, A. 2002, ApJ, 573, 138

Klaas, U., Nielbock, M., Haas, M., Krause, O., \& Schreiber, J. 2010, A\&A, 518, L44

Larsen, S. S. 2010, Royal Society of London Philosophical Transactions Series A, 368, 867

Larsen, S. S., \& Richtler, T. 2004, A\&A, 427, 495

Larson, R. B. 1981, MNRAS, 194, 809
Lupton, R., Blanton, M. R., Fekete, G., Hogg, D. W., O'Mullane, W., Szalay, A., \& Wherry, N. 2004, PASP, 116, 133

Maoz, D., Barth, A. J., Sternberg, A., Filippenko, A. V., Ho, L. C., Macchetto, F. D., Rix, H.-W., \& Schneider, D. P. 1996, AJ, 111,2248

McCrady, N., \& Graham, J. R. 2007, ApJ, 663, 844

Melo, V. P., Muñoz-Tuñón, C., Maíz-Apellániz, J., \& Tenorio-Tagle, G. 2005, ApJ, 619, 270

Mengel, S., Lehnert, M. D., Thatte, N., \& Genzel, R. 2002, A\&A, 383,137

- 2005, A\&A, 443, 41

Meurer, G. R., Heckman, T. M., Leitherer, C., Kinney, A., Robert, C., \& Garnett, D. R. 1995, AJ, 110, 2665

Mirabel, I. F., et al. 1998, A\&A, 333, L1

Neff, S. G., \& Ulvestad, J. S. 2000, AJ, 120, 670

Oka, T., Hasegawa, T., Hayashi, M., Handa, T., \& Sakamoto, S. 1998, ApJ, 493, 730

Oka, T., Hasegawa, T., Sato, F., Tsuboi, M., Miyazaki, A., \& Sugimoto, M. 2001, ApJ, 562, 348

Pahre, M. A., Ashby, M. L. N., Fazio, G. G., \& Willner, S. P. 2004, ApJS, 154, 235

Pineda, J. E., Rosolowsky, E. W., \& Goodman, A. A. 2009, ApJ, 699, L134

Portegies Zwart, S. F., McMillan, S. L. W., \& Gieles, M. 2010, ARA\&A, 48, 431

Renaud, F., Boily, C. M., Naab, T., \& Theis, C. 2009, ApJ, 706, 67

Sault, R. J., Teuben, P. J., \& Wright, M. C. H. 1995, in Astronomical Society of the Pacific Conference Series, Vol. 77, Astronomical Data Analysis Software and Systems IV, ed. R. A. Shaw, H. E. Payne, \& J. J. E. Hayes, 433

Sawada, T., et al. 2001, ApJS, 136, 189

Schulz, A., Henkel, C., Muders, D., Mao, R. Q., Röllig, M., \& Mauersberger, R. 2007, A\&A, 466, 467

Schweizer, F., et al. 2008, AJ, 136, 1482

Sheth, K., Vogel, S. N., Wilson, C. D., \& Dame, T. M. 2008, ApJ, 675,330

Shu, F. H. 1977, ApJ, 214, 488

Snijders, L., Kewley, L. J., \& van der Werf, P. P. 2007, ApJ, 669, 269

Solomon, P. M., Downes, D., \& Radford, S. J. E. 1992, ApJ, 398, L29

Solomon, P. M., Downes, D., Radford, S. J. E., \& Barrett, J. W. 1997, ApJ, 478, 144

Solomon, P. M., Rivolo, A. R., Barrett, J., \& Yahil, A. 1987, ApJ, 319,730

Ueda, J., et al. 2012, ApJ, 745, 65

van den Bergh, S. 2001, ApJ, 559, L113

Wang, Z., et al. 2004, ApJS, 154, 193

Whitmore, B. C. 2003, in A Decade of Hubble Space Telescope Science, ed. M. Livio, K. Noll, \& M. Stiavelli, 153-178

Whitmore, B. C., \& Schweizer, F. 1995, AJ, 109, 960 
Whitmore, B. C., Zhang, Q., Leitherer, C., Fall, S. M., Schweizer, F., \& Miller, B. W. 1999, AJ, 118, 1551

Whitmore, B. C., et al. 2010, AJ, 140, 75

Williams, J. P., de Geus, E. J., \& Blitz, L. 1994, ApJ, 428, 693

Wilson, C. D., Scoville, N., Madden, S. C., \& Charmandaris, V. 2000, ApJ, 542, 120

-. 2003, ApJ, 599, 1049

Wu, J., Evans, II, N. J., Gao, Y., Solomon, P. M., Shirley, Y. L., \& Vanden Bout, P. A. 2005, ApJ, 635, L173

Zepf, S. E., Ashman, K. M., English, J., Freeman, K. C., \& Sharples, R. M. 1999, AJ, 118, 752
Zezas, A., \& Fabbiano, G. 2002, ApJ, 577, 726

Zezas, A., Fabbiano, G., Rots, A. H., \& Murray, S. S. 2002, ApJ, 577,710

Zhang, H.-X., Gao, Y., \& Kong, X. 2010, MNRAS, 401, 1839

Zhang, Q., \& Fall, S. M. 1999, ApJ, 527, L81

Zhang, Q., Fall, S. M., \& Whitmore, B. C. 2001, ApJ, 561, 727

Zhu, M., Seaquist, E. R., \& Kuno, N. 2003, ApJ, 588, 243 
TABLE 2

Cloud Catalog

\begin{tabular}{|c|c|c|c|c|c|c|}
\hline & $\alpha_{2000}, \delta_{2000}$ & $\begin{array}{c}v_{\mathrm{rad}} \\
\left(\mathrm{km} \mathrm{s}^{-1}\right)\end{array}$ & $\begin{array}{c}r \\
(\mathrm{pc})\end{array}$ & $\begin{array}{c}\sigma_{v} \\
\left(\mathrm{~km} \mathrm{~s}^{-1}\right)\end{array}$ & $\begin{array}{l}\log M_{\mathrm{mol}} \\
\left(\log M_{\odot}\right)\end{array}$ & $\begin{array}{l}\log M_{\mathrm{vir}} \\
\left(\log M_{\odot}\right)\end{array}$ \\
\hline 1 & $12^{\mathrm{h}} 01^{\mathrm{m}} 53^{\mathrm{s}} .22,-18^{\circ} 53^{\prime} 09^{\prime \prime} 5$ & 1615.7 & 251 & 16.5 & 8.2 & 7.7 \\
\hline 2 & $12^{\mathrm{h}} 01^{\mathrm{m}} 55^{\mathrm{s}} .50,-18^{\circ} 52^{\prime} 47^{\prime \prime} 9$ & 1508.4 & 290 & 17.3 & 8.2 & 7.8 \\
\hline 3 & $12^{\mathrm{h}} 01^{\mathrm{m}} 54^{\mathrm{s}} .95,-18^{\circ} 53^{\prime} 05^{\prime \prime} 6$ & 1493.8 & 183 & 20.6 & 8.2 & 7.7 \\
\hline 4 & $12^{\mathrm{h}} 01^{\mathrm{m}} 55^{\mathrm{s}} .38,-18^{\circ} 52^{\prime} 48^{\prime \prime} 5$ & 1430.4 & 163 & 17.2 & 7.9 & 7.5 \\
\hline 5 & $12^{\mathrm{h}} 01^{\mathrm{m}} 53^{\mathrm{s}} .18,-18^{\circ} 53^{\prime} 09^{\prime \prime} 5$ & 1645.0 & 278 & 14.8 & 8.1 & 7.6 \\
\hline 6 & $12^{\mathrm{h}} 01^{\mathrm{m}} 54^{\mathrm{s}} .89,-18^{\circ} 52^{\prime} 51^{\prime \prime} .8$ & 1449.9 & 273 & 16.1 & 8.1 & 7.7 \\
\hline 7 & $12^{\mathrm{h}} 01^{\mathrm{m}} 54^{\mathrm{s}} .91,-18^{\circ} 52^{\prime} 52^{\prime \prime} 4$ & 1469.4 & 311 & 15.6 & 8.1 & 7.7 \\
\hline 8 & $12^{\mathrm{h}} 01^{\mathrm{m}} 55^{\mathrm{s}} .38,-18^{\circ} 52^{\prime} 48^{\prime \prime} 2$ & 1391.4 & 282 & 17.6 & 8.2 & 7.8 \\
\hline 9 & $12^{\mathrm{h}} 01^{\mathrm{m}} 54^{\mathrm{s}} .93,-18^{\circ} 53^{\prime} 02^{\prime \prime} 3$ & 1557.2 & 317 & 22.5 & 8.4 & 8.1 \\
\hline 10 & $12^{\mathrm{h}} 01^{\mathrm{m}} 53^{\mathrm{s}} .56,-18^{\circ} 53^{\prime} 08^{\prime \prime} 9$ & 1596.2 & 184 & 15.7 & 7.9 & 7.5 \\
\hline 11 & $12^{\mathrm{h}} 01^{\mathrm{m}} 55^{\mathrm{s}} \cdot 19,-18^{\circ} 52^{\prime} 40^{\prime \prime} \cdot 7$ & 1576.7 & 275 & 12.3 & 7.9 & 7.5 \\
\hline 12 & $12^{\mathrm{h}} 01^{\mathrm{m}} 53^{\mathrm{s}} .58,-18^{\circ} 53^{\prime} 08^{\prime \prime} 9$ & 1610.9 & 142 & 8.6 & 7.4 & 6.9 \\
\hline 13 & $12^{\mathrm{h}} 01^{\mathrm{m}} 53^{\mathrm{s}} .56,-18^{\circ} 53^{\prime} 09^{\prime \prime} .2$ & 1625.5 & 209 & 25.4 & 8.0 & 8.0 \\
\hline 14 & $12^{\mathrm{h}} 01^{\mathrm{m}} 54^{\mathrm{s}} \cdot 66,-18^{\circ} 53^{\prime} 05^{\prime \prime} 0$ & 1474.3 & 293 & 13.1 & 8.0 & 7.5 \\
\hline 15 & $12^{\mathrm{h}} 01^{\mathrm{m}} 55^{\mathrm{s}} .48,-18^{\circ} 52^{\prime} 46^{\prime \prime} 4$ & 1459.7 & 240 & 25.6 & 8.0 & 8.0 \\
\hline 16 & $12^{\mathrm{h}} 01^{\mathrm{m}} 54^{\mathrm{s}} .21,-18^{\circ} 53^{\prime} 08^{\prime \prime} 0$ & 1425.5 & 226 & 17.7 & 7.7 & 7.7 \\
\hline 17 & $12^{\mathrm{h}} 01^{\mathrm{m}} 53^{\mathrm{s}} .12,-18^{\circ} 53^{\prime} 09^{\prime \prime} 8$ & 1703.5 & 241 & 16.5 & 7.8 & 7.7 \\
\hline 18 & $12^{\mathrm{h}} 01^{\mathrm{m}} 54^{\mathrm{s}} .79,-18^{\circ} 52^{\prime} 54^{\prime \prime} 5$ & 1610.9 & 149 & 17.9 & 7.6 & 7.5 \\
\hline 19 & $12^{\mathrm{h}} 01^{\mathrm{m}} 54^{\mathrm{s}} .93,-18^{\circ} 52^{\prime} 58^{\prime \prime} 7$ & 1493.8 & 262 & 19.2 & 7.6 & 7.8 \\
\hline 20 & $12^{\mathrm{h}} 01^{\mathrm{m}} 54^{\mathrm{s}} .76,-18^{\circ} 52^{\prime} 55^{\prime \prime} \cdot 7$ & 1645.0 & 183 & 14.9 & 7.6 & 7.5 \\
\hline 21 & $12^{\mathrm{h}} 01^{\mathrm{m}} 53^{\mathrm{s}} .20,-18^{\circ} 53^{\prime} 16^{\prime \prime} \cdot 7$ & 1727.9 & 224 & 11.9 & 7.5 & 7.3 \\
\hline 22 & $12^{\mathrm{h}} 01^{\mathrm{m}} 55^{\mathrm{s}} \cdot 40,-18^{\circ} 53^{\prime} 02^{\prime \prime} 0$ & 1518.2 & 168 & 10.4 & 7.4 & 7.1 \\
\hline 23 & $12^{\mathrm{h}} 01^{\mathrm{m}} 53^{\mathrm{s}} .22,-18^{\circ} 53^{\prime} 16^{\prime \prime} 1$ & 1708.4 & 249 & 9.1 & 7.4 & 7.2 \\
\hline 24 & $12^{\mathrm{h}} 01^{\mathrm{m}} 54^{\mathrm{s}} \cdot 36,-18^{\circ} 53^{\prime} 08^{\prime \prime} 0$ & 1454.8 & 170 & 11.6 & 7.5 & 7.2 \\
\hline 25 & $12^{\mathrm{h}} 01^{\mathrm{m}} 54^{\mathrm{s}} .89,-18^{\circ} 53^{\prime} 06^{\prime \prime} \cdot 2$ & 1610.9 & 244 & 20.5 & 7.7 & 7.9 \\
\hline 26 & $12^{\mathrm{h}} 01^{\mathrm{m}} 54^{\mathrm{s}} .45,-18^{\circ} 52^{\prime} 49^{\prime \prime} \cdot 4$ & 1479.2 & 118 & 12.2 & 7.1 & 7.1 \\
\hline 27 & $12^{\mathrm{h}} 01^{\mathrm{m}} 54^{\mathrm{s}} \cdot 60,-18^{\circ} 52^{\prime} 45^{\prime \prime} 5$ & 1601.1 & 225 & 9.9 & 7.4 & 7.2 \\
\hline 28 & $12^{\mathrm{h}} 01^{\mathrm{m}} 53^{\mathrm{s}} \cdot 20,-18^{\circ} 53^{\prime} 16^{\prime \prime} 4$ & 1679.1 & 266 & 16.4 & 7.5 & 7.7 \\
\hline 29 & $12^{\mathrm{h}} 01^{\mathrm{m}} 54^{\mathrm{s}} .45,-18^{\circ} 52^{\prime} 44^{\prime \prime} 9$ & 1620.6 & 161 & 12.4 & 7.2 & 7.2 \\
\hline 30 & $12^{\mathrm{h}} 01^{\mathrm{m}} 55^{\mathrm{s}} .00,-18^{\circ} 52^{\prime} 53^{\prime \prime} 0$ & 1562.1 & 89 & 10.1 & 7.0 & 6.8 \\
\hline 31 & $12^{\mathrm{h}} 01^{\mathrm{m}} 53^{\mathrm{s}} \cdot 66,-18^{\circ} 53^{\prime} 12^{\prime \prime} 2$ & 1571.8 & 141 & 7.7 & 7.0 & 6.8 \\
\hline 32 & $12^{\mathrm{h}} 01^{\mathrm{m}} 53^{\mathrm{s}} \cdot 64,-18^{\circ} 53^{\prime} 11^{\prime \prime} 9$ & 1581.6 & 119 & 11.9 & 7.0 & 7.1 \\
\hline 33 & $12^{\mathrm{h}} 01^{\mathrm{m}} 54^{\mathrm{s}} .89,-18^{\circ} 52^{\prime} 45^{\prime \prime} 5$ & 1381.6 & 118 & 9.1 & 7.0 & 6.8 \\
\hline 34 & $12^{\mathrm{h}} 01^{\mathrm{m}} 55^{\mathrm{s}} \cdot 65,-18^{\circ} 52^{\prime} 51^{\prime \prime} 5$ & 1537.7 & 79 & 5.6 & 6.7 & 6.2 \\
\hline 35 & $12^{\mathrm{h}} 01^{\mathrm{m}} 53^{\mathrm{s}} .16,-18^{\circ} 53^{\prime} 20^{\prime \prime} 9$ & 1762.0 & 113 & 9.5 & 7.0 & 6.9 \\
\hline 36 & $12^{\mathrm{h}} 01^{\mathrm{m}} 54^{\mathrm{s}} .55,-18^{\circ} 52^{\prime} 45^{\prime \prime} \cdot 2$ & 1615.7 & 94 & 12.9 & 7.0 & 7.0 \\
\hline 37 & $12^{\mathrm{h}} 01^{\mathrm{m}} 54^{\mathrm{s}} .91,-18^{\circ} 53^{\prime} 07^{\prime \prime} 1$ & 1645.0 & 216 & 10.4 & 7.3 & 7.2 \\
\hline 38 & $12^{\mathrm{h}} 01^{\mathrm{m}} 53^{\mathrm{s}} .92,-18^{\circ} 53^{\prime} 18^{\prime \prime} 2$ & 1703.5 & 211 & 7.7 & 7.0 & 6.9 \\
\hline 39 & $12^{\mathrm{h}} 01^{\mathrm{m}} 54^{\mathrm{s}} \cdot 40,-18^{\circ} 52^{\prime} 49^{\prime \prime} \cdot 7$ & 1488.9 & 55 & 10.7 & 6.9 & 6.6 \\
\hline 40 & $12^{\mathrm{h}} 01^{\mathrm{m}} 54^{\mathrm{s}} .87,-18^{\circ} 52^{\prime} 59^{\prime \prime} 6$ & 1610.9 & 204 & 14.4 & 7.2 & 7.5 \\
\hline 41 & $12^{\mathrm{h}} 01^{\mathrm{m}} 54^{\mathrm{s}} .81,-18^{\circ} 52^{\prime} 47^{\prime \prime} \cdot 6$ & 1386.5 & 261 & 18.9 & 7.4 & 7.8 \\
\hline 42 & $12^{\mathrm{h}} 01^{\mathrm{m}} 54^{\mathrm{s}} .11,-18^{\circ} 53^{\prime} 26^{\prime \prime} 3$ & 1606.0 & 53 & 4.2 & 6.4 & 5.8 \\
\hline 43 & $12^{\mathrm{h}} 01^{\mathrm{m}} 55^{\mathrm{s}} .23,-18^{\circ} 53^{\prime} 09^{\prime \prime} 5$ & 1630.4 & 168 & 8.7 & 6.8 & 6.9 \\
\hline 44 & $12^{\mathrm{h}} 01^{\mathrm{m}} 54^{\mathrm{s}} .00,-18^{\circ} 53^{\prime} 09^{\prime \prime} 5$ & 1498.7 & 70 & 7.3 & 6.7 & 6.4 \\
\hline 45 & $12^{\mathrm{h}} 01^{\mathrm{m}} 54^{\mathrm{s}} .57,-18^{\circ} 52^{\prime} 46^{\prime \prime} 4$ & 1430.4 & 229 & 11.7 & 7.2 & 7.3 \\
\hline 46 & $12^{\mathrm{h}} 01^{\mathrm{m}} 55^{\mathrm{s}} .25,-18^{\circ} 53^{\prime} 09^{\prime \prime} 2$ & 1640.1 & 75 & 7.2 & 6.6 & 6.4 \\
\hline 47 & $12^{\mathrm{h}} 01^{\mathrm{m}} 54^{\mathrm{s}} \cdot 74,-18^{\circ} 52^{\prime} 40^{\prime \prime} 4$ & 1513.3 & $<80$ & 12.1 & 6.6 & $<6.9$ \\
\hline 48 & $12^{\mathrm{h}} 01^{\mathrm{m}} 54^{\mathrm{s}} .28,-18^{\circ} 52^{\prime} 50^{\prime \prime} 3$ & 1532.8 & 134 & 11.9 & 6.9 & 7.1 \\
\hline 49 & $12^{\mathrm{h}} 01^{\mathrm{m}} 54^{\mathrm{s}} .55,-18^{\circ} 52^{\prime} 45^{\prime \prime} 8$ & 1396.3 & 71 & 5.9 & 6.5 & 6.2 \\
\hline 50 & $12^{\mathrm{h}} 01^{\mathrm{m}} 55^{\mathrm{s}} .33,-18^{\circ} 52^{\prime} 43^{\prime \prime} 7$ & 1645.0 & 176 & 12.0 & 6.8 & 7.2 \\
\hline 51 & $12^{\mathrm{h}} 01^{\mathrm{m}} 54^{\mathrm{s}} .76,-18^{\circ} 52^{\prime} 53^{\prime \prime} 3$ & 1527.9 & 102 & 16.0 & 6.8 & 7.3 \\
\hline 52 & $12^{\mathrm{h}} 01^{\mathrm{m}} 55^{\mathrm{s}} .48,-18^{\circ} 53^{\prime} 07^{\prime \prime} 4$ & 1601.1 & 149 & 10.0 & 6.8 & 7.0 \\
\hline 53 & $12^{\mathrm{h}} 01^{\mathrm{m}} 53^{\mathrm{s}} \cdot 66,-18^{\circ} 53^{\prime} 12^{\prime \prime} 8$ & 1552.3 & 97 & 7.0 & 6.5 & 6.5 \\
\hline 54 & $12^{\mathrm{h}} 01^{\mathrm{m}} 55^{\mathrm{s}} .02,-18^{\circ} 52^{\prime} 52^{\prime \prime} 7$ & 1552.3 & 227 & 18.1 & 7.1 & 7.7 \\
\hline 55 & $12^{\mathrm{h}} 01^{\mathrm{m}} 54^{\mathrm{s}} \cdot 17,-18^{\circ} 52^{\prime} 47^{\prime \prime} \cdot 3$ & 1513.3 & 109 & 7.1 & 6.6 & 6.6 \\
\hline 56 & $12^{\mathrm{h}} 01^{\mathrm{m}} 55^{\mathrm{s}} .25,-18^{\circ} 52^{\prime} 43^{\prime \prime} 1$ & 1537.7 & 114 & 10.0 & 6.6 & 6.9 \\
\hline 57 & $12^{\mathrm{h}} 01^{\mathrm{m}} 54^{\mathrm{s}} .26,-18^{\circ} 52^{\prime} 49^{\prime \prime} 4$ & 1523.1 & 127 & 12.8 & 6.8 & 7.2 \\
\hline 58 & $12^{\mathrm{h}} 01^{\mathrm{m}} 54^{\mathrm{s}} .17,-18^{\circ} 52^{\prime} 45^{\prime \prime} 8$ & 1615.7 & $<80$ & 2.1 & 6.3 & $<5.4$ \\
\hline 59 & $12^{\mathrm{h}} 01^{\mathrm{m}} 54^{\mathrm{s}} \cdot 49,-18^{\circ} 52^{\prime} 45^{\prime \prime} 2$ & 1406.0 & 128 & 10.6 & 6.6 & 7.0 \\
\hline 60 & $12^{\mathrm{h}} 01^{\mathrm{m}} 53^{\mathrm{s}} \cdot 64,-18^{\circ} 53^{\prime} 08^{\prime \prime} 9$ & 1527.9 & 238 & 11.8 & 7.1 & 7.4 \\
\hline 61 & $12^{\mathrm{h}} 01^{\mathrm{m}} 54^{\mathrm{s}} .19,-18^{\circ} 52^{\prime} 44^{\prime \prime} 6$ & 1610.9 & $<80$ & 9.4 & 6.3 & $<6.7$ \\
\hline 62 & $12^{\mathrm{h}} 01^{\mathrm{m}} 53^{\mathrm{s}} .94,-18^{\circ} 53^{\prime} 17^{\prime \prime} 9$ & 1684.0 & 220 & 6.2 & 6.8 & 6.8 \\
\hline 63 & $12^{\mathrm{h}} 01^{\mathrm{m}} 54^{\mathrm{s}} \cdot 66,-18^{\circ} 52^{\prime} 41^{\prime \prime} 3$ & 1518.2 & 35 & 7.4 & 6.6 & 6.1 \\
\hline 64 & $12^{\mathrm{h}} 01^{\mathrm{m}} 53^{\mathrm{s}} .28,-18^{\circ} 53^{\prime} 21^{\prime \prime} 8$ & 1737.7 & $<80$ & 11.2 & 6.3 & $<6.8$ \\
\hline 65 & $12^{\mathrm{h}} 01^{\mathrm{m}} 55^{\mathrm{s}} \cdot 65,-18^{\circ} 52^{\prime} 41^{\prime \prime} 9$ & 1606.0 & 144 & 11.0 & 6.5 & 7.1 \\
\hline 66 & $12^{\mathrm{h}} 01^{\mathrm{m}} 54^{\mathrm{s}} .38,-18^{\circ} 52^{\prime} 47^{\prime \prime} 3$ & 1527.9 & 163 & 13.9 & 6.9 & 7.3 \\
\hline
\end{tabular}


TABLE 2 - Continued

\begin{tabular}{|c|c|c|c|c|c|c|}
\hline & $\alpha_{2000}, \delta_{2000}$ & $\begin{array}{c}v_{\mathrm{rad}} \\
\left(\mathrm{km} \mathrm{s}^{-1}\right)\end{array}$ & $\begin{array}{c}r \\
(\mathrm{pc})\end{array}$ & $\begin{array}{c}\sigma_{v} \\
\left(\mathrm{~km} \mathrm{~s}^{-1}\right)\end{array}$ & $\begin{array}{l}\log M_{\text {mol }} \\
\left(\log M_{\odot}\right)\end{array}$ & $\begin{array}{l}\log M_{\mathrm{vir}} \\
\left(\log M_{\odot}\right)\end{array}$ \\
\hline 67 & $12^{\mathrm{h}} 01^{\mathrm{m}} 53^{\mathrm{s}} \cdot 24,-18^{\circ} 53^{\prime} 17^{\prime \prime} 0$ & 1762.0 & 189 & 10.4 & 6.8 & 7.2 \\
\hline 68 & $12^{\mathrm{h}} 01^{\mathrm{m}} 53^{\mathrm{s}} \cdot 69,-18^{\circ} 53^{\prime} 13^{\prime \prime} \cdot 4$ & 1645.0 & 166 & 24.0 & 6.8 & 7.8 \\
\hline 69 & $12^{\mathrm{h}} 01^{\mathrm{m}} 54^{\mathrm{s}} .17,-18^{\circ} 52^{\prime} 45^{\prime \prime} .^{\prime}$ & 1601.1 & $<80$ & 6.3 & 6.1 & $<6.3$ \\
\hline 70 & $12^{\mathrm{h}} 01^{\mathrm{m}} 54^{\mathrm{s}} .72,-18^{\circ} 52^{\prime} 53^{\prime \prime} .^{\prime}$ & 1547.5 & 99 & 10.7 & 6.7 & 6.9 \\
\hline 71 & $12^{\mathrm{h}} 01^{\mathrm{m}} 54^{\mathrm{s}} \cdot 13,-18^{\circ} 52^{\prime} 47^{\prime \prime} \cdot 3$ & 1459.7 & $<80$ & $<4.9$ & 5.4 & $<6.1$ \\
\hline 72 & $12^{\mathrm{h}} 01^{\mathrm{m}} 54^{\mathrm{s}} .17,-18^{\circ} 53^{\prime} 17^{\prime \prime} 0$ & 1532.8 & 130 & 9.0 & 6.5 & 6.9 \\
\hline 73 & $12^{\mathrm{h}} 01^{\mathrm{m}} 55^{\mathrm{s}} .50,-18^{\circ} 52^{\prime} 52^{\prime \prime}{ }^{\prime \prime} 4$ & 1474.3 & $<80$ & 1.6 & 5.9 & $<5.1$ \\
\hline 74 & $12^{\mathrm{h}} 01^{\mathrm{m}} 53^{\mathrm{s}} .07,-18^{\circ} 53^{\prime} 18^{\prime \prime} \cdot 2$ & 1445.0 & $<80$ & $<4.9$ & 5.6 & $<6.1$ \\
\hline 75 & $12^{\mathrm{h}} 01^{\mathrm{m}} 55^{\mathrm{s}} \cdot 59,-18^{\circ} 52^{\prime} 53^{\prime \prime} \cdot 3$ & 1493.8 & $<80$ & 2.0 & 5.9 & $<5.3$ \\
\hline 76 & $12^{\mathrm{h}} 01^{\mathrm{m}} 55^{\mathrm{s}} \cdot 50,-18^{\circ} 52^{\prime} 52^{\prime \prime} \cdot 7$ & 1440.2 & $<80$ & 3.5 & 5.9 & $<5.8$ \\
\hline 77 & $12^{\mathrm{h}} 01^{\mathrm{m}} 53^{\mathrm{s}} .73,-18^{\circ} 53^{\prime} 13^{\prime \prime} .^{\prime}$ & 1664.5 & $<80$ & 2.0 & 5.8 & $<5.4$ \\
\hline 78 & $12^{\mathrm{h}} 01^{\mathrm{m}} 54^{\mathrm{s}} \cdot 21,-18^{\circ} 52^{\prime} 43^{\prime \prime} \cdot 7$ & 1415.8 & $<80$ & $<4.9$ & 5.2 & $<6.1$ \\
\hline 79 & $12^{\mathrm{h}} 01^{\mathrm{m}} 53^{\mathrm{s}} \cdot 60,-18^{\circ} 53^{\prime} 17^{\prime \prime} \cdot 6$ & 1430.4 & $<80$ & $<4.9$ & 5.5 & $<6.1$ \\
\hline 80 & $12^{\mathrm{h}} 01^{\mathrm{m}} 54^{\mathrm{s}} .05,-18^{\circ} 53^{\prime} 18^{\prime \prime} \cdot 2$ & 1498.7 & $<80$ & 4.2 & 5.9 & $<6.0$ \\
\hline 81 & $12^{\mathrm{h}} 01^{\mathrm{m}} 55^{\mathrm{s}} \cdot 38,-18^{\circ} 53^{\prime} 05^{\prime \prime} 6$ & 1567.0 & $<80$ & 2.5 & 5.9 & $<5.5$ \\
\hline 82 & $12^{\mathrm{h}} 01^{\mathrm{m}} 54^{\mathrm{s}} .30,-18^{\circ} 52^{\prime} 41^{\prime \prime} 9$ & 1518.2 & $<80$ & 1.2 & 5.7 & $<4.9$ \\
\hline 83 & $12^{\mathrm{h}} 01^{\mathrm{m}} 53^{\mathrm{s}} .16,-18^{\circ} 53^{\prime} 09^{\prime \prime} 5$ & 1479.2 & 105 & $<4.9$ & 6.1 & $<6.2$ \\
\hline 84 & $12^{\mathrm{h}} 01^{\mathrm{m}} 54^{\mathrm{s}} .26,-18^{\circ} 52^{\prime} 54^{\prime \prime} \cdot 2$ & 1659.6 & $<80$ & $<4.9$ & 5.4 & $<6.1$ \\
\hline 85 & $12^{\mathrm{h}} 01^{\mathrm{m}} 54^{\mathrm{s}} .40,-18^{\circ} 52^{\prime} 44^{\prime \prime} \cdot 3$ & 1376.8 & $<80$ & $<4.9$ & 5.8 & $<6.1$ \\
\hline 86 & $12^{\mathrm{h}} 01^{\mathrm{m}} 55^{\mathrm{s}} \cdot 61,-18^{\circ} 52^{\prime} 51^{\prime \prime} 5$ & 1508.4 & $<80$ & $<4.9$ & 5.4 & $<6.1$ \\
\hline 87 & $12^{\mathrm{h}} 01^{\mathrm{m}} 53^{\mathrm{s}} .18,-18^{\circ} 53^{\prime} 15^{\prime \prime} 5$ & 1396.3 & $<80$ & $<4.9$ & 5.3 & $<6.1$ \\
\hline 88 & $12^{\mathrm{h}} 01^{\mathrm{m}} 54^{\mathrm{s}} .53,-18^{\circ} 53^{\prime} 24^{\prime \prime}{ }^{\prime} 8$ & 1513.3 & 41 & 2.4 & 6.0 & 5.2 \\
\hline 89 & $12^{\mathrm{h}} 01^{\mathrm{m}} 55^{\mathrm{s}} \cdot 23,-18^{\circ} 52^{\prime} 41^{\prime \prime} .^{\prime}$ & 1615.7 & $<80$ & $<4.9$ & 5.2 & $<6.1$ \\
\hline 90 & $12^{\mathrm{h}} 01^{\mathrm{m}} 53^{\mathrm{s}} .69,-18^{\circ} 53^{\prime} 10^{\prime \prime} 7$ & 1488.9 & $<80$ & $<4.9$ & 5.5 & $<6.1$ \\
\hline 91 & $12^{\mathrm{h}} 01^{\mathrm{m}} 54^{\mathrm{s}} \cdot 24,-18^{\circ} 52^{\prime} 45^{\prime \prime} \cdot 2$ & 1367.0 & $<80$ & $<4.9$ & 5.3 & $<6.1$ \\
\hline 92 & $12^{\mathrm{h}} 01^{\mathrm{m}} 54^{\mathrm{s}} .53,-18^{\circ} 53^{\prime} 17^{\prime \prime} \cdot 3$ & 1674.3 & $<80$ & 1.8 & 5.7 & $<5.2$ \\
\hline 93 & $12^{\mathrm{h}} 01^{\mathrm{m}} 54^{\mathrm{s}} .11,-18^{\circ} 53^{\prime} 26^{\prime \prime} 6$ & 1615.7 & $<80$ & $<4.9$ & 5.4 & $<6.1$ \\
\hline 94 & $12^{\mathrm{h}} 01^{\mathrm{m}} 53^{\mathrm{s}} .88,-18^{\circ} 53^{\prime} 08^{\prime \prime} \cdot 3$ & 1479.2 & $<80$ & 0.8 & 5.6 & $<4.5$ \\
\hline 95 & $12^{\mathrm{h}} 01^{\mathrm{m}} 54^{\mathrm{s}} .85,-18^{\circ} 53^{\prime} 16^{\prime \prime} 1$ & 1664.5 & $<80$ & $<4.9$ & 5.5 & $<6.1$ \\
\hline 96 & $12^{\mathrm{h}} 01^{\mathrm{m}} 53^{\mathrm{s}} .71,-18^{\circ} 53^{\prime} 13^{\prime \prime} \cdot 7$ & 1527.9 & $<80$ & $<4.9$ & 5.2 & $<6.1$ \\
\hline 97 & $12^{\mathrm{h}} 01^{\mathrm{m}} 53^{\mathrm{s}} \cdot 20,-18^{\circ} 53^{\prime} 08^{\prime \prime} \cdot 3$ & 1464.5 & $<80$ & $<4.9$ & 5.6 & $<6.1$ \\
\hline 98 & $12^{\mathrm{h}} 01^{\mathrm{m}} 53^{\mathrm{s}} .58,-18^{\circ} 53^{\prime} 10^{\prime \prime} 1$ & 1732.8 & $<80$ & $<4.9$ & 5.1 & $<6.1$ \\
\hline 99 & $12^{\mathrm{h}} 01^{\mathrm{m}} 54^{\mathrm{s}} .11,-18^{\circ} 52^{\prime} 49^{\prime \prime} 1$ & 1606.0 & $<80$ & $<4.9$ & 5.3 & $<6.1$ \\
\hline 100 & $12^{\mathrm{h}} 01^{\mathrm{m}} 54^{\mathrm{s}} \cdot 11,-18^{\circ} 53^{\prime} 26^{\prime \prime} 3$ & 1649.9 & $<80$ & 1.0 & 5.4 & $<4.7$ \\
\hline 101 & $12^{\mathrm{h}} 01^{\mathrm{m}} 54^{\mathrm{s}} .34,-18^{\circ} 52^{\prime} 47^{\prime \prime} 9$ & 1567.0 & $<80$ & $<4.9$ & 5.6 & $<6.1$ \\
\hline 102 & $12^{\mathrm{h}} 01^{\mathrm{m}} 54^{\mathrm{s}} .19,-18^{\circ} 53^{\prime} 18^{\prime \prime} 5$ & 1503.6 & $<80$ & $<4.9$ & 5.3 & $<6.1$ \\
\hline 103 & $12^{\mathrm{h}} 01^{\mathrm{m}} 54^{\mathrm{s}} .91,-18^{\circ} 52^{\prime} 38^{\prime \prime} \cdot 9$ & 1328.0 & $<80$ & $<4.9$ & 5.1 & $<6.1$ \\
\hline 104 & $12^{\mathrm{h}} 01^{\mathrm{m}} 53^{\mathrm{s}} \cdot 79,-18^{\circ} 53^{\prime} 20^{\prime \prime} \cdot 6$ & 1484.1 & $<80$ & 4.5 & 5.7 & $<6.0$ \\
\hline 105 & $12^{\mathrm{h}} 01^{\mathrm{m}} 53^{\mathrm{s}} .73,-18^{\circ} 53^{\prime} 19^{\prime \prime} 7$ & 1727.9 & $<80$ & $<4.9$ & 5.1 & $<6.1$ \\
\hline 106 & $12^{\mathrm{h}} 01^{\mathrm{m}} 54^{\mathrm{s}} .13,-18^{\circ} 53^{\prime} 26^{\prime \prime} 9$ & 1635.2 & $<80$ & $<4.9$ & 4.9 & $<6.1$ \\
\hline 107 & $12^{\mathrm{h}} 01^{\mathrm{m}} 54^{\mathrm{s}} \cdot 28,-18^{\circ} 52^{\prime} 51^{\prime \prime} .5$ & 1669.4 & $<80$ & $<4.9$ & 5.3 & $<6.1$ \\
\hline 108 & $12^{\mathrm{h}} 01^{\mathrm{m}} 54^{\mathrm{s}} .21,-18^{\circ} 53^{\prime} 11^{\prime \prime} 6$ & 1537.7 & $<80$ & $<4.9$ & 5.2 & $<6.1$ \\
\hline 109 & $12^{\mathrm{h}} 01^{\mathrm{m}} 54^{\mathrm{s}} .17,-18^{\circ} 52^{\prime} 49^{\prime \prime} \cdot 7$ & 1479.2 & $<80$ & $<4.9$ & 5.3 & $<6.1$ \\
\hline 110 & $12^{\mathrm{h}} 01^{\mathrm{m}} 55^{\mathrm{s}} .44,-18^{\circ} 52^{\prime} 45^{\prime \prime} 8$ & 1645.0 & $<80$ & $<4.9$ & 5.4 & $<6.1$ \\
\hline 111 & $12^{\mathrm{h}} 01^{\mathrm{m}} 53^{\mathrm{s}} \cdot 22,-18^{\circ} 53^{\prime} 12^{\prime \prime} .^{\prime}$ & 1488.9 & $<80$ & $<4.9$ & 5.0 & $<6.1$ \\
\hline 112 & $12^{\mathrm{h}} 01^{\mathrm{m}} 54^{\mathrm{s}} .02,-18^{\circ} 53^{\prime} 16^{\prime \prime} .4$ & 1742.5 & $<80$ & $<4.9$ & 5.5 & $<6.1$ \\
\hline 113 & $12^{\mathrm{h}} 01^{\mathrm{m}} 54^{\mathrm{s}} \cdot 21,-18^{\circ} 53^{\prime} 21^{\prime \prime} 8$ & 1732.8 & $<80$ & $<4.9$ & 5.2 & $<6.1$ \\
\hline 114 & $12^{\mathrm{h}} 01^{\mathrm{m}} 54^{\mathrm{s}} \cdot 28,-18^{\circ} 52^{\prime} 43^{\prime \prime} .^{\prime}$ & 1537.7 & $<80$ & $<4.9$ & 5.1 & $<6.1$ \\
\hline 115 & $12^{\mathrm{h}} 01^{\mathrm{m}} 54^{\mathrm{s}} .72,-18^{\circ} 53^{\prime} 05^{\prime \prime} \cdot 9$ & 1654.8 & $<80$ & $<4.9$ & 5.5 & $<6.1$ \\
\hline 116 & $12^{\mathrm{h}} 01^{\mathrm{m}} 53^{\mathrm{s}} \cdot 18,-18^{\circ} 53^{\prime} 09^{\prime \prime} \cdot 2$ & 1493.8 & $<80$ & 1.3 & 5.3 & $<4.9$ \\
\hline 117 & $12^{\mathrm{h}} 01^{\mathrm{m}} 54^{\mathrm{s}} \cdot 62,-18^{\circ} 53^{\prime} 08^{\prime \prime} .^{\prime} 0$ & 1381.6 & $<80$ & $<4.9$ & 5.0 & $<6.1$ \\
\hline 118 & $12^{\mathrm{h}} 01^{\mathrm{m}} 53^{\mathrm{s}} .88,-18^{\circ} 53^{\prime} 11^{\prime \prime} 6$ & 1527.9 & $<80$ & $<4.9$ & 5.0 & $<6.1$ \\
\hline 119 & $12^{\mathrm{h}} 01^{\mathrm{m}} 54^{\mathrm{s}} .26,-18^{\circ} 52^{\prime} 44^{\prime \prime} .^{\prime} 0$ & 1547.5 & $<80$ & 1.0 & 4.9 & $<4.8$ \\
\hline 120 & $12^{\mathrm{h}} 01^{\mathrm{m}} 54^{\mathrm{s}} .98,-18^{\circ} 52^{\prime} 44^{\prime \prime} 3$ & 1645.0 & $<80$ & $<4.9$ & 5.3 & $<6.1$ \\
\hline 121 & $12^{\mathrm{h}} 01^{\mathrm{m}} 53^{\mathrm{s}} .43,-18^{\circ} 53^{\prime} 18^{\prime \prime} .^{\prime} 8$ & 1391.4 & $<80$ & $<4.9$ & 4.9 & $<6.1$ \\
\hline 122 & $12^{\mathrm{h}} 01^{\mathrm{m}} 53^{\mathrm{s}} \cdot 69,-18^{\circ} 53^{\prime} 17^{\prime \prime} \cdot 6$ & 1718.2 & $<80$ & $<4.9$ & 4.9 & $<6.1$ \\
\hline 123 & $12^{\mathrm{h}} 01^{\mathrm{m}} 55^{\mathrm{s}} .48,-18^{\circ} 52^{\prime} 45^{\prime \prime} 5$ & 1620.6 & $<80$ & $<4.9$ & 5.1 & $<6.1$ \\
\hline 124 & $12^{\mathrm{h}} 01^{\mathrm{m}} 54^{\mathrm{s}} .19,-18^{\circ} 52^{\prime} 47^{\prime \prime} 6$ & 1674.3 & $<80$ & $<4.9$ & 4.9 & $<6.1$ \\
\hline 125 & $12^{\mathrm{h}} 01^{\mathrm{m}} 53^{\mathrm{s}} .54,-18^{\circ} 53^{\prime} 18^{\prime \prime} \cdot 2$ & 1786.4 & $<80$ & $<4.9$ & 4.9 & $<6.1$ \\
\hline 126 & $12^{\mathrm{h}} 01^{\mathrm{m}} 55^{\mathrm{s}} \cdot 61,-18^{\circ} 52^{\prime} 43^{\prime \prime} \cdot 7$ & 1586.5 & $<80$ & $<4.9$ & 4.9 & $<6.1$ \\
\hline 127 & $12^{\mathrm{h}} 01^{\mathrm{m}} 54^{\mathrm{s}} .11,-18^{\circ} 53^{\prime} 26^{\prime \prime} 9$ & 1537.7 & $<80$ & $<4.9$ & 4.9 & $<6.1$ \\
\hline 128 & $12^{\mathrm{h}} 01^{\mathrm{m}} 54^{\mathrm{s}} \cdot 28,-18^{\circ} 53^{\prime} 16^{\prime \prime} 1$ & 1562.1 & $<80$ & $<4.9$ & 4.9 & $<6.1$ \\
\hline 129 & $12^{\mathrm{h}} 01^{\mathrm{m}} 53^{\mathrm{s}} .92,-18^{\circ} 53^{\prime} 06^{\prime \prime} 5$ & 1513.3 & $<80$ & $<4.9$ & 4.9 & $<6.1$ \\
\hline 130 & $12^{\mathrm{h}} 01^{\mathrm{m}} 54^{\mathrm{s}} .09,-18^{\circ} 53^{\prime} 25^{\prime \prime} .^{\prime}$ & 1635.2 & $<80$ & $<4.9$ & 5.1 & $<6.1$ \\
\hline 131 & $12^{\mathrm{h}} 01^{\mathrm{m}} 54^{\mathrm{s}} .15,-18^{\circ} 53^{\prime} 10^{\prime \prime} \cdot 7$ & 1640.1 & $<80$ & $<4.9$ & 5.0 & $<6.1$ \\
\hline 132 & $12^{\mathrm{h}} 01^{\mathrm{m}} 54^{\mathrm{s}} .74,-18^{\circ} 53^{\prime} 05^{\prime \prime} \cdot 9$ & 1674.3 & $<80$ & 1.3 & 4.9 & $<4.9$ \\
\hline
\end{tabular}


TABLE $2-$ Continued

\begin{tabular}{cccccc}
\hline \hline$\alpha_{2000}, \delta_{2000}$ & $\begin{array}{c}v_{\text {rad }} \\
\left(\mathrm{km} \mathrm{s}^{-1}\right)\end{array}$ & $\begin{array}{c}r \\
(\mathrm{pc})\end{array}$ & $\begin{array}{c}\sigma_{v} \\
\left(\mathrm{~km} \mathrm{~s}^{-1}\right)\end{array}$ & $\begin{array}{c}\log M_{\operatorname{mol}} \\
\left(\log M_{\odot}\right)\end{array}$ & $\begin{array}{c}\log M_{\text {vir }} \\
\left(\log M_{\odot}\right)\end{array}$ \\
\hline
\end{tabular}

Note. - Basic cloud properties from clumpfind. Clouds that were unresolved spatially or in velocity are assigned upper limits of 80 pc and $4.9 \mathrm{~km} \mathrm{~s}^{-1}$, respectively. Note that the velocity is defined using the radio definition $(v=c z /(1+z))$. 